The Quarterly Journal of Mathematics Advance Access published on August 6, 2007

Quart. J. Math. 58 (2007), 415-441; doi:10.1093/qmath/ham002

\title{
GLUING TECHNIQUES IN TRIANGULAR GEOMETRY
}

\author{
by PAUL BALMER ${ }^{\dagger}$ \\ (Department Mathematics, ETH Zurich, 8092 Zurich, Switzerland) \\ and GIORDANO FAVI ${ }^{\ddagger}$ \\ (Department Mathematics, Universität Basel, 4051 Basel, Switzerland)
}

[Received 7 December 2006]

\begin{abstract}
We discuss gluing of objects and gluing of morphisms in triangulated categories. We illustrate the results by producing, among other things, a Mayer-Vietoris exact sequence involving Picard groups.
\end{abstract}

\section{Introduction}

Tensor triangular geometry is the geometry of tensor triangulated categories. Heuristically, this contains at least algebraic geometry and the geometry of modular representation theory but it also appears in many other areas of mathematics, as recalled in [1, Introduction].

We will denote by $\mathcal{K}$ a triangulated category (with suspension $T: \mathcal{K} \stackrel{\sim}{\rightarrow} \mathcal{K}$ ) equipped with a tensor product, that is, an exact symmetric monoidal structure $\otimes: \mathcal{K} \times \mathcal{K} \longrightarrow \mathcal{K}$; see more in section 1 . Two key examples to keep in mind appear, respectively, in algebraic geometry, as $\mathcal{K}=\mathrm{D}^{\text {perf }}(X)$, the derived category of perfect complexes over a quasi-compact and quasi-separated scheme $X$ (for example, a noetherian scheme), and in modular representation theory, as $\mathcal{K}=k G-$ stab, the stable category of finite dimensional representations modulo projective ones, for $G$ a finite group and $k$ a field of characteristic $p>0$, typically dividing the order of the group.

In [1], the spectrum, $\operatorname{Spc}(\mathcal{K})$, of such categories is introduced. It is the universal topological space in which one can define supports, $\operatorname{supp}(a) \subset \operatorname{Spc}(\mathcal{K})$, for objects $a \in \mathcal{K}$ in a reasonable way. In the above two examples, this spectrum is, respectively, isomorphic to the scheme $X$ itself and to the projective support variety $\operatorname{Proj} \mathrm{H}^{\bullet}(G, k)$.

One fundamental construction of [1] is the presheaf of triangulated categories, $U \mapsto \mathcal{K}(U)$, which associates to an open $U \subset \operatorname{Spc}(\mathcal{K})$ a tensor triangulated category $\mathcal{K}(U)$ defined as follows. Consider $Z=\operatorname{Spc}(\mathcal{K}) \backslash U$ the closed complement of $U$ and consider the thick subcategory $\mathcal{K}_{Z} \subset \mathcal{K}$ of those objects $a \in \mathcal{K}$ with $\operatorname{supp}(a) \subset Z$, that is, those objects which ought to disappear on $U$. Then, the category

$$
\mathcal{K}(U):=\widetilde{\mathcal{K} / \mathcal{K}_{Z}}
$$

is defined as the idempotent completion of the Verdier quotient $\mathcal{K} / \mathcal{K}_{Z}$. Localization $\mathcal{K} \rightarrow \mathcal{K} / \mathcal{K}_{Z}$ followed by idempotent completion $\mathcal{K} / \mathcal{K}_{Z} \hookrightarrow \mathcal{K}(U)$ yields a restriction functor $\rho_{U}: \mathcal{K} \rightarrow \mathcal{K}(U)$. In the scheme example, it is an important theorem of Thomason [10] that for a quasi-compact open

\footnotetext{
†Corresponding author. E-mail: balmer@math.ethz.ch

E-mail: giordano.favi@unibas.ch
} 
$U \subset X$ and for $\mathcal{K}=\mathrm{D}^{\text {perf }}(X)$, the above $\mathcal{K}(U)$ is equivalent to $\mathrm{D}^{\text {perf }}(U)$. This is one reason for working with idempotent complete categories. Another reason is a key result of [2] which says that if $\mathcal{K}$ is idempotent complete and if the support of an object of $\mathcal{K}$ decomposes into two connected components then the object itself decomposes into two direct summands accordingly, see Theorem 1.8 below.

The present paper deals with the following type of questions. Suppose that $\operatorname{Spc}(\mathcal{K})$ is covered by two quasi-compact open subsets $\operatorname{Spc}(\mathcal{K})=U_{1} \cup U_{2}$ and consider the commutative diagram of restriction functors:

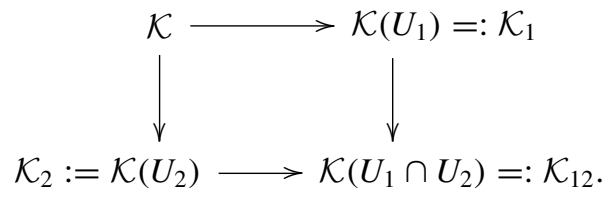

QUESTION Is the global category $\mathcal{K}$ obtained by 'gluing' $\mathcal{K}_{1}$ and $\mathcal{K}_{2}$ over $\mathcal{K}_{12}$ ?

This is a very natural question but it is known to be tricky, already in algebraic geometry. Indeed, it is easy to find non-zero morphisms $f: a \rightarrow b$ in $\mathcal{K}=\mathrm{D}^{\text {perf }}(X)$ such that $f_{\left.\right|_{U_{1}}}=0$ and $f_{\left.\right|_{U_{2}}}=0$ for an open cover $X=U_{1} \cup U_{2}$. Over $X=\mathbb{P}_{k}^{1}$ an example is the morphism $f: \mathcal{O}(2) \rightarrow T(\mathcal{O})$ which is the third one in the exact triangle associated to the non-split Koszul exact sequence $\mathcal{O} \longmapsto \mathcal{O}(1) \oplus$ $\mathcal{O}(1) \rightarrow \mathcal{O}(2)$; take for $U_{1}$ and $U_{2}$ two affine subsets. (For an exact sequence of vector bundles $E^{\prime} \longmapsto E \rightarrow E^{\prime \prime}$ over a scheme $X$, the corresponding morphism $f: E^{\prime \prime} \rightarrow T\left(E^{\prime}\right)$ is zero in $\mathrm{D}^{\text {perf }}(X)$ if and only if the sequence splits.) This example also shows that the phenomenon is not pathological but observable in very common situations.

Still, the problem admits the following solution, to be found in our main results:

THEOREM (Mayer-Vietoris for morphisms, see Corollary 5.8) In the above situation (1), given two objects $a, b \in \mathcal{K}$, there exists a long exact sequence:

$$
\cdots \operatorname{Hom}_{\mathcal{K}_{12}}(T a, b) \stackrel{\partial}{\rightarrow} \operatorname{Hom}_{\mathcal{K}}(a, b) \rightarrow \operatorname{Hom}_{\mathcal{K}_{1}}(a, b) \oplus \operatorname{Hom}_{\mathcal{K}_{2}}(a, b) \rightarrow \operatorname{Hom}_{\mathcal{K}_{12}}(a, b) \stackrel{\partial}{\rightarrow} \cdots
$$

The connecting homomorphism $\partial: \operatorname{Hom}_{\mathcal{K}_{12}}(T a, b) \rightarrow \operatorname{Hom}_{\mathcal{K}}(a, b)$ is defined in Construction 3.1. The other homomorphisms are the obvious restrictions and differences thereof.

THEOREM (Gluing of two objects, see Corollary 5.10) In the above situation (1), given two objects $a_{1} \in \mathcal{K}_{1}$ and $a_{2} \in \mathcal{K}_{2}$ and an isomorphism $\sigma: a_{1} \stackrel{\sim}{\rightarrow} a_{2}$ in $\mathcal{K}_{12}$, there exists an object $a \in \mathcal{K}$ which becomes isomorphic to $a_{i}$ in $\mathcal{K}_{i}$ for $i=1,2$. Moreover, this gluing is unique up to (possibly nonunique) isomorphism.

We can extend the above result to three open subsets and three objects, at the cost of possibly losing uniqueness of the gluing:

COROLLARY (Gluing of three objects, see Corollary 5.11) Let $\operatorname{Spc}(\mathcal{K})=U_{1} \cup U_{2} \cup U_{3}$ be a cover by quasi-compact open subsets. Consider three objects $a_{i} \in \mathcal{K}\left(U_{i}\right)$ for $i=1,2,3$ and three isomorphisms $\sigma_{i j}: a_{j} \stackrel{\sim}{\rightarrow} a_{i}$ in $\mathcal{K}\left(U_{i} \cap U_{j}\right)$ for $1 \leq i<j \leq 3$. Suppose that the cocycle relation $\sigma_{12} \circ \sigma_{23}=\sigma_{13}$ is satisfied in $\mathcal{K}\left(U_{1} \cap U_{2} \cap U_{3}\right)$. Then there exists an object $a \in \mathcal{K}$, isomorphic to $a_{i}$ in $\mathcal{K}\left(U_{i}\right)$ for $i=1,2,3$. 
In general, we do not know if this gluing is possible with more than three open subsets. Nevertheless, in Theorem 5.13, we give elementary conditions under which the gluing is possible for arbitrary covers.

Of course, there is a gigantic literature on Mayer-Vietoris long exact sequences. We do not doubt that the reader considers such ideas as basic mathematics and does not expect us to survey former Mayer-Vietoris results here. Let us simply mention, in the framework of algebraic geometry, that our results recover Thomason's Mayer-Vietoris long exact sequences for $\mathrm{D}^{\text {perf }}(X)$, see [9], which themselves already generalized Mayer-Vietoris long exact sequences in Zariski cohomology.

We then apply the above main results to obtain an exact sequence involving Picard groups. For us, the Picard group, $\operatorname{Pic}(\mathcal{K})$, is the set of isomorphism classes of invertible objects in $\mathcal{K}$, with the tensor product as multiplication. In algebraic geometry, $\operatorname{Pic}\left(D^{\text {perf }}(X)\right)$ is well known to be the usual Picard group of $X$ up to possible shifts, see Proposition 6.4. On the other hand, $\operatorname{Pic}(k G-\mathrm{stab})$ is nothing but the group of endotrivial representations, which is one of the fundamental invariants of modular representation theory. In the next statement, we denote by $\mathbb{G}_{\mathrm{m}}(\mathcal{K})=\operatorname{Hom}_{\mathcal{K}}(\mathbf{1}, \mathbf{1})^{\times}$the abelian group of automorphisms of the $\otimes$-unit object $\mathbf{1} \in \mathcal{K}$.

TheOREM (Mayer-Vietoris for Picard groups, see Theorem 6.7) Let $\operatorname{Spc}(\mathcal{K})=U_{1} \cup U_{2}$ with $U_{i}$ quasi-compact. See (1). Then there is half a long exact sequence:

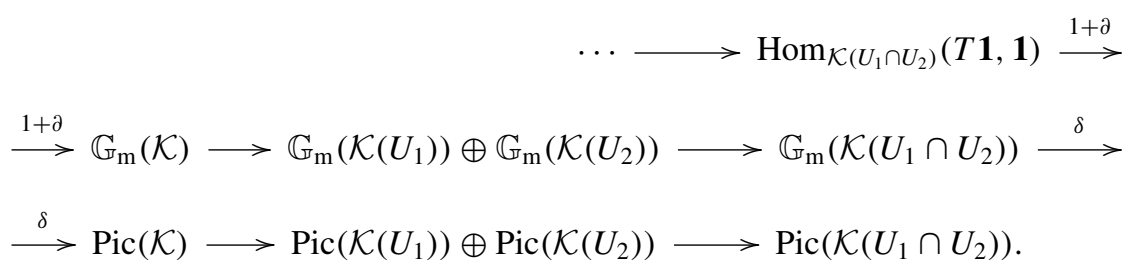

To the left, we have the Mayer-Vietoris long exact sequence, the homomorphism $\partial$ is as before and the non-labelled morphisms are again the obvious restrictions and (multiplicative) differences of restrictions. The new homomorphism

$$
\delta: \mathbb{G}_{\mathrm{m}}\left(\mathcal{K}\left(U_{1} \cap U_{2}\right)\right) \rightarrow \operatorname{Pic}(\mathcal{K})
$$

assigns to a unit $\sigma \in \mathbb{G}_{\mathrm{m}}\left(\mathcal{K}\left(U_{1} \cap U_{2}\right)\right)$ the invertible object obtained by gluing two copies of the objects $\mathbf{1} \in \mathcal{K}\left(U_{1}\right)$ and $\mathbf{1} \in \mathcal{K}\left(U_{2}\right)$ along $\sigma: \mathbf{1} \stackrel{\sim}{\rightarrow} \mathbf{1}$ in $\mathcal{K}\left(U_{1} \cap U_{2}\right)$.

It would be very interesting to continue this sequence to the right, say, with Brauer groups of Azumaya algebras. Although this is still work in progress, the authors do not know yet whether such an extension is possible in general. Neither do we know what the Brauer group of $\mathcal{K}=k G-$ stab should be, for instance.

In fact, in modular representation theory, applying the above results to $\mathcal{K}=k G-$ stab gives us a way to construct endotrivial $k G$-modules from any Čech $\mathbb{G}_{\mathrm{m}}$-cocycle over the projective support variety $\operatorname{Proj} \mathrm{H}^{\bullet}(G, k)$, as long as the involved cover has at most three open pieces. In particular, the map $\delta$ of the last result might be of interest to representation theorists and we do not know if it has been studied, even in special cases. Dave Benson and Jon Carlson suggested a possible link with the recent article [5]. This is investigated in a subsequent paper [3]. 
Using the conditional gluing of more than three objects, we obtain the following result (Theorem 6.8), which relates invertible modules over the spectrum $\operatorname{Spc}(\mathcal{K})$ and invertible objects in $\mathcal{K}$. See more comments in Remark 6.9.

THEOREM Suppose that $\operatorname{Hom}_{\mathcal{K}(U)}(T \mathbf{1}, \mathbf{1})=0$ for every quasi-compact open subsets $U \subset \operatorname{Spc}(\mathcal{K})$. Then, gluing induces an injective homomorphism from the first Čech cohomology of $\operatorname{Spc}(\mathcal{K})$ with coefficients in $\mathbb{G}_{\mathrm{m}}$ into the Picard group of $\mathcal{K}$

$$
\check{\mathrm{H}}^{1}\left(\operatorname{Spc}(\mathcal{K}), \mathbb{G}_{\mathrm{m}}\right) \hookrightarrow \operatorname{Pic}(\mathcal{K})
$$

For completeness, we give in section 7 the following variant of Mayer-Vietoris:

TheOREM (Excision, see Theorem 7.1) Let $Y \subset U \subset \operatorname{Spc}(\mathcal{K})$. Assume that $Y$ is closed with quasicompact complement and that $U$ is open and quasi-compact. Then the restriction functor $\mathcal{K} \rightarrow \mathcal{K}(U)$ induces an equivalence between the subcategories of objects supported on $Y$, that is, $\mathcal{K}_{Y} \stackrel{\sim}{\longrightarrow} \mathcal{K}(U)_{Y}$.

The referee of the first version of this article suggested we give more formal proofs, postponing as long as possible the assumption that $\mathcal{K}$ carries a tensor product. We therefore rewrote the paper with the 'formal Mayer-Vietoris' language of sections 2-4. These sections can be read without reference to tensor triangular geometry, that is, without assuming $\mathcal{K}$ has a tensor structure. Some readers will benefit from this gain of generality, despite a little loss in geometric intuition. Then, tensor triangular geometry really enters the game in section 5 .

\section{Recalling tensor triangular geometry}

We survey the main concepts and results of [1] and [2]. Standard notions about triangulated categories can be found in Verdier [11] or Neeman [8].

Definitions 1.1 A tensor triangulated category $(\mathcal{K}, \otimes, \mathbf{1})$ is an essentially small triangulated category $\mathcal{K}$ with a symmetric monoidal structure $\otimes: \mathcal{K} \times \mathcal{K} \longrightarrow \mathcal{K},(a, b) \mapsto a \otimes b$. We have in particular $a \otimes b \cong b \otimes a$ and $\mathbf{1} \otimes a \cong a$ for the unit $\mathbf{1} \in \mathcal{K}$. We assume moreover that the functors $a \otimes-$ and $-\otimes b$ are exact for every $a, b \in \mathcal{K}$ and that the usual diagram

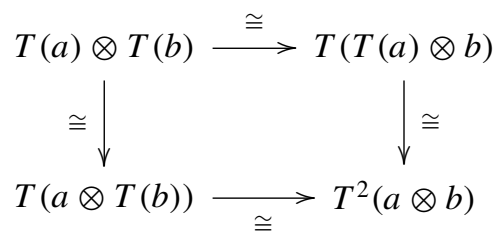

anti-commutes. We use $T: \mathcal{K} \stackrel{\sim}{\rightarrow} \mathcal{K}$ to denote the translation (suspension).

A prime ideal $\mathcal{P} \subsetneq \mathcal{K}$ is a proper subcategory such that (1)-(4) below hold true:

(1) $\mathcal{P}$ is a full triangulated subcategory, that is, $0 \in \mathcal{P}, T(\mathcal{P})=\mathcal{P}$ and if $a, b \in \mathcal{P}$ and if $a \rightarrow$ $b \rightarrow c \rightarrow T(a)$ is a distinguished triangle in $\mathcal{K}$ then $c \in \mathcal{P}$; 
(2) $\mathcal{P}$ is thick, that is, if $a \oplus b \in \mathcal{P}$ then $a, b \in \mathcal{P}$;

(3) $\mathcal{P}$ is a $\otimes$-ideal, that is, if $a \in \mathcal{P}$ then $a \otimes b \in \mathcal{P}$ for all $b \in \mathcal{K}$;

(4) $\mathcal{P}$ is prime, that is, if $a \otimes b \in \mathcal{P}$ then $a \in \mathcal{P}$ or $b \in \mathcal{P}$.

A subcategory $\mathcal{J} \subset \mathcal{K}$ satisfying (1), (2) and (3) is called a thick $\otimes$-ideal.

The spectrum $\operatorname{Spc}(\mathcal{K})$ is the set of primes $\mathcal{P} \subset \mathcal{K}$. The support of an object $a \in \mathcal{K}$ is defined as the subset $\operatorname{supp}(a)=\{\mathcal{P} \in \operatorname{Spc}(\mathcal{K}) \mid a \notin \mathcal{P}\} \subset \operatorname{Spc}(\mathcal{K})$. The complements $\mathrm{U}(a)=\{\mathcal{P} \in \operatorname{Spc}(\mathcal{K}) \mid a \in$ $\mathcal{P}\}$ of these supports form a basis $\{\mathrm{U}(a)\}_{a \in \mathcal{K}}$ of the so-called Zariski topology on the spectrum.

THEOREM 1.2 [1, Theorem 3.2] Let $\mathcal{K}$ be a tensor triangulated category. We have

(i) $\operatorname{supp}(0)=\varnothing$ and $\operatorname{supp}(\mathbf{1})=\operatorname{Spc}(\mathcal{K})$.

(ii) $\operatorname{supp}(a \oplus b)=\operatorname{supp}(a) \cup \operatorname{supp}(b)$.

(iii) $\operatorname{supp}(T a)=\operatorname{supp}(a)$.

(iv) $\operatorname{supp}(a) \subset \operatorname{supp}(b) \cup \operatorname{supp}(c)$ for any distinguished $a \rightarrow b \rightarrow c \rightarrow T(a)$.

(v) $\operatorname{supp}(a \otimes b)=\operatorname{supp}(a) \cap \operatorname{supp}(b)$.

Moreover, $(\operatorname{Spc}(\mathcal{K}), \operatorname{supp})$ is universal for these properties.

Notation 1.3 Let $Y \subset \operatorname{Spc}(\mathcal{K})$. We denote by $\mathcal{K}_{Y}$ the full subcategory $\mathcal{K}_{Y}:=\{a \in \mathcal{K} \mid \operatorname{supp}(a) \subset Y\}$ of those objects supported on $Y$.

Definition 1.4 We call a tensor triangulated category $(\mathcal{K}, \otimes, \mathbf{1})$ strongly closed if there exists a bi-exact functor hom : $\mathcal{K}^{\mathrm{op}} \times \mathcal{K} \longrightarrow \mathcal{K}$ with natural isomorphisms

$$
\operatorname{Hom}_{\mathcal{K}}(a \otimes b, c) \cong \operatorname{Hom}_{\mathcal{K}}(a, \operatorname{hom}(b, c))
$$

and such that all objects are strongly dualizable, that is, the natural morphism

$$
D(a) \otimes b \stackrel{\sim}{\rightarrow} \operatorname{hom}(a, b)
$$

is an isomorphism for all $a, b \in \mathcal{K}$, where we denote by $D(a)$ the dual $D(a):=\operatorname{hom}(a, \mathbf{1})$ of an object $a \in \mathcal{K}$. More details can be found in [7, Appendix A], for instance. It follows from (3) that $D^{2}(a) \cong a$ for all $a \in \mathcal{K}$; see for instance [7, Theorem A.2.5(b)].

PROPOSITION 1.5 [2, Corollary 2.5] Let $\mathcal{K}$ be a strongly closed tensor triangulated category and let $a \in \mathcal{K}$ be an object. Then $\operatorname{supp}(a)=\varnothing$ if and only if $a=0$.

PROPOSITION 1.6 [2, Corollary 2.8] Let $\mathcal{K}$ be a strongly closed tensor triangulated category. Suppose that the supports of two objects do not meet: $\operatorname{supp}(a) \cap \operatorname{supp}(b)=\varnothing$. Then there is no non-trivial morphism between them: $\operatorname{Hom}_{\mathcal{K}}(a, b)=0$.

PROPOSITION 1.7 Let $\mathcal{K}$ be a strongly closed tensor triangulated category. A morphism $f: a \rightarrow b$ in $\mathcal{K}$ is an isomorphism if and only if it is an isomorphism in $\mathcal{K} / \mathcal{P}$ for all $\mathcal{P} \in \operatorname{Spc}(\mathcal{K})$.

Proof. This easily follows from the fact that a morphism $f$ in a triangulated category is an isomorphism if and only if cone $(f)=0$. Thus, if $f$ is an isomorphism in $\mathcal{K} / \mathcal{P}$ we have that cone $(f) \in \mathcal{P}$. 
If this is true for all $\mathcal{P} \in \operatorname{Spc}(\mathcal{K})$ we have that $\operatorname{supp}(\operatorname{cone}(f))=\varnothing$ which implies that cone $(f)=0$ by Proposition 1.5.

THEOREM 1.8 [2, Theorem 2.11] Let $\mathcal{K}$ be a strongly closed tensor triangulated category. Assume that $\mathcal{K}$ is idempotent complete. Then, if the support of an object $a \in \mathcal{K}$ can be decomposed as $\operatorname{supp}(a)=Y_{1} \cup Y_{2}$ for disjoint closed subsets $Y_{1}, Y_{2} \subset \operatorname{Spc}(\mathcal{K})$, with each open complement $\operatorname{Spc}(\mathcal{K}) \backslash Y_{i}$ quasi-compact, then the object itself can be decomposed as a direct sum $a \simeq a_{1} \oplus a_{2}$ with $\operatorname{supp}\left(a_{i}\right)=Y_{i}$ for $i=1,2$.

REMARK 1.9 Recall that an additive category $\mathcal{K}$ is idempotent complete (or pseudo-abelian or karoubian) if all idempotents of all objects split, that is, if $e \in \operatorname{Hom}_{\mathcal{K}}(a, a)$ with $e^{2}=e$ then the object $a$ decomposes as a direct sum $a \simeq a^{\prime} \oplus a^{\prime \prime}$ on which $e$ becomes $\left(\begin{array}{ll}1 & 0 \\ 0 & 0\end{array}\right)$, that is, $a \cong$ $\operatorname{Im}(e) \oplus \operatorname{Ker}(e)$. One can always 'idempotent complete' an additive category $\mathcal{K} \hookrightarrow \widetilde{\mathcal{K}}$. If $\mathcal{K}$ is triangulated, its idempotent completion $\widetilde{\mathcal{K}}$ inherits a unique triangulation such that $\mathcal{K} \hookrightarrow \widetilde{\mathcal{K}}$ is exact, see more in [4].

The rest of the paper heavily relies on the next set of definitions:

Definitions 1.10 Let $\mathcal{K}$ be an idempotent complete strongly closed tensor triangulated category. Let $U$ be a quasi-compact open subset of $\operatorname{Spc}(\mathcal{K})$, and let us denote by $Z=\operatorname{Spc}(\mathcal{K}) \backslash$ $U$ its closed complement. We denote by $\mathcal{L}(U)=\mathcal{K} / \mathcal{K}_{Z}$ the Verdier localization with respect to $\mathcal{K}_{Z}$ (which can be realized by keeping the same objects as $\mathcal{K}$ and by inverting all morphisms whose cone belongs to $\mathcal{K}_{Z}$, by means of calculus of fractions). We denote by $\mathcal{K}(U)=$ $\widetilde{\mathcal{L}(U)}$ its idempotent completion. We have a fully faithful cofinal morphism $\mathcal{L}(U) \hookrightarrow \mathcal{K}(U)$. (Some authors say dense instead of cofinal, like in [9]. This means that every object $a$ of the big category is a direct summand of an object of the small one, for instance $a \oplus T a$. See [4].)

For $U=\operatorname{Spc}(\mathcal{K})$, by Proposition 1.5, we have $\mathcal{L}(U)=\mathcal{K}=\widetilde{\mathcal{K}}=\mathcal{K}(U)$ since we assume $\mathcal{K}$ idempotent complete. If $U \subset V$ we denote by $\rho_{U, V}: \mathcal{L}(V) \rightarrow \mathcal{L}(U)$ the localization functor and we also denote by

$$
\rho_{U, V}: \mathcal{K}(V) \rightarrow \mathcal{K}(U)
$$

the induced functor, that we call the restriction functor from $V$ to $U$. When $V=\operatorname{Spc}(\mathcal{K})$, we simply write $\rho_{U}: \mathcal{K} \rightarrow \mathcal{K}(U)$ for $\rho_{U, V}$.

For two objects $a, b$ of $\mathcal{K}$ we denote by

$$
\operatorname{Hom}_{\mathcal{K}(U)}(a, b):=\operatorname{Hom}_{\mathcal{L}(U)}\left(\rho_{U}(a), \rho_{U}(b)\right)=\operatorname{Hom}_{\mathcal{K}(U)}\left(\rho_{U}(a), \rho_{U}(b)\right)
$$

the set of morphisms between $\rho_{U}(a)$ and $\rho_{U}(b)$ in $\mathcal{L}(U)$ or equivalently in its idempotent completion $\mathcal{K}(U)$; for simplicity, we might speak of morphisms between $a$ and $b$ in $\mathcal{K}(U)$, or simply morphisms between $a$ and $b$ over $U$.

Proposition 1.11 For $U \subset \operatorname{Spc}(\mathcal{K})$ quasi-compact and open, the restriction functor $\rho_{U}: \mathcal{K} \rightarrow \mathcal{K}(U)$ induces a homeomorphism $\operatorname{Spc}(\mathcal{K}(U)) \stackrel{\sim}{\rightarrow} U$, under which $\operatorname{supp}\left(\rho_{U}(a)\right)=U \cap \operatorname{supp}(a)$, for any object $a \in \mathcal{K}$. 
Proof. In fact, by [1, Corollary 3.14], $\operatorname{Spc}(\mathcal{K}(U))=\operatorname{Spc}\left(\mathcal{K} / \mathcal{K}_{Z}\right)$ and by loc. cit. Proposition 3.11, the localization functor induces a homeomorphism between $\operatorname{Spc}\left(\mathcal{K} / \mathcal{K}_{Z}\right)$ and the subspace $V:=\{\mathcal{P} \in$ $\left.\operatorname{Spc}(\mathcal{K}) \mid \mathcal{K}_{Z} \subset \mathcal{P}\right\}$ of $\operatorname{Spc}(\mathcal{K})$. So, it suffices to check that $V=U$. The last equality $\operatorname{supp}\left(\rho_{U}(a)\right)=$ $U \cap \operatorname{supp}(a)$ will then be a general fact about the functor $\operatorname{Spc}(-)$, see loc. cit. Proposition 3.6.

Let $\mathcal{P} \in \operatorname{Spc}(\mathcal{K})$. By the classification of thick $\otimes$-ideals, loc. cit. [1, Theorem 4.10], we have $\mathcal{P} \in V$, that is, $\mathcal{K}_{Z} \subset \mathcal{P}$, if and only if $Z=\operatorname{supp}\left(\mathcal{K}_{Z}\right) \subset \operatorname{supp}(\mathcal{P}) \stackrel{\text { def. }}{=} \cup_{a \in \mathcal{P}} \operatorname{supp}(a)$. By taking complements, this is equivalent to $\cap_{a \in \mathcal{P}} U(a) \subset U$, where $U(a)=\operatorname{Spc}(\mathcal{K}) \backslash \operatorname{supp}(a)=\{\mathcal{Q} \in$ $\operatorname{Spc}(\mathcal{K}) \mid a \in \mathcal{Q}\}$. Tautologically, $\cap_{a \in \mathcal{P}} U(a)=\{\mathcal{Q} \in \operatorname{Spc}(\mathcal{K}) \mid \mathcal{P} \subset \mathcal{Q}\}$. The latter set is contained in $U$ if and only if $\mathcal{P} \in U$ : one direction is trivial and the other one uses that $Z$ is specialization closed, see loc. cit. [1, Proposition 2.9]. So, $\mathcal{P} \in V$ if and only if $\mathcal{P} \in U$, as was left to check.

REMARK 1.12 The above result cannot hold without assuming $U$ quasi-compact since $\operatorname{Spc}(\mathcal{K})$ is quasi-compact for any $\mathcal{K}$. It is used above to insure $Z=\operatorname{supp}\left(\mathcal{K}_{Z}\right)$.

We end this section with some general facts about triangulated categories.

LEMMA 1.13 Let $\mathcal{K}$ be a triangulated category. Then for every distinguished triangle in which one object decomposes into two direct summands

$$
a \stackrel{s}{\longrightarrow} b \stackrel{\left(\begin{array}{l}
\alpha \\
\beta
\end{array}\right)}{\longrightarrow} c_{1} \oplus c_{2} \stackrel{(\gamma \delta}{\longrightarrow} T a
$$

there exist two objects, $d$ and e, and four distinguished triangles:

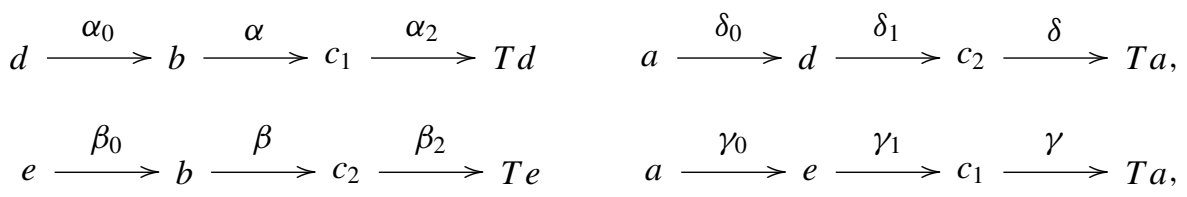

where $\alpha_{2}=T \delta_{0} \gamma, \delta_{1}=\beta \alpha_{0}, \beta_{2}=T \gamma_{0} \delta$ and $\gamma_{1}=\alpha \beta_{0}$. Moreover, the morphism s factors as $s=$ $\alpha_{0} \delta_{0}=\beta_{0} \gamma_{0}$.

In particular, we have cone $(\alpha) \simeq \operatorname{cone}(\delta)$ and cone $(\beta) \simeq \operatorname{cone}(\gamma)$.

Proof. We will prove the existence of the first two triangles, the other two are obtained symmetrically $\left(c_{1} \oplus c_{2} \simeq c_{2} \oplus c_{1}\right)$. The triangles are obtained by applying the Octahedron Axiom to the equality 
$\left(\begin{array}{ll}1 & 0\end{array}\right)\left(\begin{array}{l}\alpha \\ \beta\end{array}\right)=\alpha$ as displayed below:

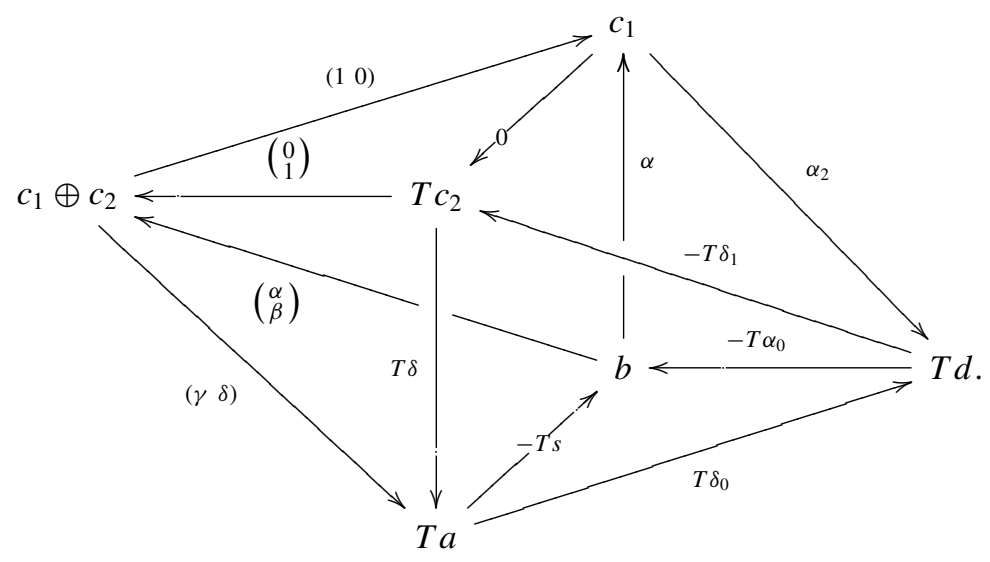

DEFINITION 1.14 We say that a commutative square as follows is a weak push-out

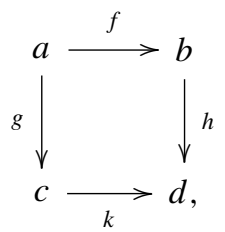

if there exists a distinguished triangle $a \stackrel{\left(\begin{array}{l}f \\ g\end{array}\right)}{\longrightarrow} b \oplus c \stackrel{(-h k)}{\longrightarrow} d \stackrel{\ell}{\longrightarrow} T a$ for some morphism $\ell: d \rightarrow T(a)$. This is justified since $(d, h, k)$ satisfies the universal property of the push-out of $f$ and $g$ but without uniqueness of the factorization. Since such a square is then also a weak pull-back, we call it weakly bicartesian.

\section{Formal Mayer-Vietoris covers}

The referee sagaciously suggested that we make clear how our proofs only depend on Theorem 1.8, which is indeed the keystone to Mayer-Vietoris. In this logic, we now give an abstract version of our results, only assuming the conclusions of Theorem 1.8, without necessarily carrying the tensor structure around.

Definition 2.1 Let $\mathcal{K}$ be an idempotent complete triangulated category. A formal Mayer-Vietoris cover of $\mathcal{K}$ is the data of two thick triangulated subcategories $\mathcal{J}_{1}$ and $\mathcal{J}_{2}$ of $\mathcal{K}$ such that $\operatorname{Hom}_{\mathcal{K}}\left(c_{1}, c_{2}\right)=\operatorname{Hom}_{\mathcal{K}}\left(c_{2}, c_{1}\right)=0$ for every pair of objects $c_{1} \in \mathcal{J}_{1}$ and $c_{2} \in \mathcal{J}_{2}$. This implies in particular that $\mathcal{J}_{1} \cap \mathcal{J}_{2}=0$. 
REMARK 2.2 The subcategories $\mathcal{J}_{1}$ and $\mathcal{J}_{2}$ do not really 'cover' $\mathcal{K}$ (for example, $\mathcal{J}_{1}=\mathcal{J}_{2}=0$ define a 'formal cover'). In fact, the cover is rather realized by the corresponding restrictions (see Definition 1.10), that is, the localization functors $\mathcal{K} \rightarrow \mathcal{K} / \mathcal{J}_{1}$ and $\mathcal{K} \rightarrow \mathcal{K} / \mathcal{J}_{2}$ followed by the idempotent completions. See Construction 2.4.

The following is a proto-Theorem 1.8.

LEMMA 2.3 Given a formal Mayer-Vietoris cover of $\mathcal{K}$, the full subcategory $\mathcal{J}_{1} \oplus \mathcal{J}_{2}$ of $\mathcal{K}$ on the objects of the form $c_{1} \oplus c_{2}$ where $c_{1} \in \mathcal{J}_{1}$ and $c_{2} \in \mathcal{J}_{2}$, is a thick triangulated subcategory of $\mathcal{K}$.

Proof. See the proof of [2, Theorem 2.11]. We sketch it for the convenience of the reader. To see that $\mathcal{J}_{1} \oplus \mathcal{J}_{2}$ is a triangulated subcategory, it suffices to show that the cone of any morphism $f$ : $c_{1} \oplus c_{2} \rightarrow d_{1} \oplus d_{2}$ with $c_{i}, d_{i} \in \mathcal{J}_{i}$ also belongs to $\mathcal{J}_{1} \oplus \mathcal{J}_{2}$. This follows from the fact that $f$ must be diagonal by assumption. To see that $\mathcal{J}_{1} \oplus \mathcal{J}_{2}$ is a thick subcategory of $\mathcal{K}$, take a direct summand of an object $c_{1} \oplus c_{2} \in \mathcal{J}_{1} \oplus \mathcal{J}_{2}$ that we can describe as the image, $\operatorname{Im}(e)$, of some (projection) idempotent $e=e^{2} \in \operatorname{End}_{\mathcal{K}}\left(c_{1} \oplus c_{2}\right)$. By assumption, $e$ must be diagonal, that is, $e=\left(\begin{array}{cc}e_{1} & 0 \\ 0 & e_{2}\end{array}\right)$ with $e_{i}=e_{i}^{2}$ on $c_{i}$. Since $\mathcal{K}$ is idempotent complete, $c_{i} \cong \operatorname{Im}\left(e_{i}\right) \oplus \operatorname{Ker}\left(e_{i}\right)$ for $i=1,2$. Since $\mathcal{J}_{i}$ is thick, $\operatorname{Im}\left(e_{i}\right) \in \mathcal{J}_{i}$ and our direct summand $\operatorname{Im}(e) \cong \operatorname{Im}\left(e_{1}\right) \oplus \operatorname{Im}\left(e_{2}\right)$ belongs to $\mathcal{J}_{1} \oplus \mathcal{J}_{2}$ as was to be shown.

Note that the above result is wrong if $\mathcal{K}$ is not idempotent complete, as shown in the counterexample [2, Example 2.13], because $\mathcal{J}_{1} \oplus \mathcal{J}_{2}$ needs not be thick in $\mathcal{K}$.

Construction 2.4 Given a formal Mayer-Vietoris cover of $\mathcal{K}$ (Definition 2.1), let us define $\mathcal{J}_{12}:=$ $\mathcal{J}_{1} \oplus \mathcal{J}_{2}$. We can consider the Verdier quotients $\mathcal{L}_{1}=\mathcal{K} / \mathcal{J}_{1}, \mathcal{L}_{2}=\mathcal{K} / \mathcal{J}_{2}, \mathcal{L}_{12}=\mathcal{K} / \mathcal{J}_{12}$ and the respective idempotent completions $\mathcal{K}_{1}=\widetilde{\mathcal{L}}_{1}, \mathcal{K}_{2}=\widetilde{\mathcal{L}}_{2}$ and $\mathcal{K}_{12}=\widetilde{\mathcal{L}}_{12}$ (see Remark 1.9). We have the following commutative diagram:

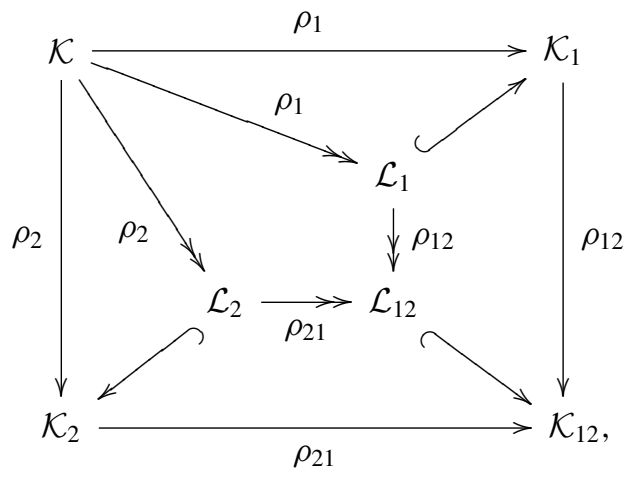

where $\rightarrow$ indicates a Verdier localization and $\hookrightarrow$ a fully faithful cofinal embedding.

REMARK 2.5 Of course, the outer square of (4) should be thought of as the formal version of the square (1) of the Introduction.

Definition 2.6 For any $i \in\{1,2,12\}$, we say that a morphism $s: a \rightarrow b$ in $\mathcal{K}$ is a $\mathcal{K}_{i}$-isomorphism if $s$ becomes an isomorphism in the localization $\mathcal{K}_{i}$ (or equivalently in $\mathcal{L}_{i}$ ). The morphism $s$ is a $\mathcal{K}_{i}$-isomorphism if and only if cone $(s) \in \mathcal{J}_{i}$. 
REMARK 2.7 In a formal Mayer-Vietoris situation, a morphism which is both a $\mathcal{K}_{1^{-}}$and a $\mathcal{K}_{2^{-}}$ isomorphism must be an isomorphism since its cone belongs to $\mathcal{J}_{1} \cap \mathcal{J}_{2}=0$.

NotATION 2.8 Like in Definitions 1.10 , for two objects $a, b \in \mathcal{K}$, we abbreviate

$$
\begin{aligned}
\operatorname{Hom}_{\mathcal{K}_{1}}(a, b) & :=\operatorname{Hom}_{\mathcal{K}_{1}}\left(\rho_{1}(a), \rho_{1}(b)\right)=\operatorname{Hom}_{\mathcal{L}_{1}}\left(\rho_{1}(a), \rho_{1}(b)\right) \\
\operatorname{Hom}_{\mathcal{K}_{2}}(a, b) & :=\operatorname{Hom}_{\mathcal{K}_{2}}\left(\rho_{2}(a), \rho_{2}(b)\right)=\operatorname{Hom}_{\mathcal{L}_{2}}\left(\rho_{2}(a), \rho_{2}(b)\right) \\
\operatorname{Hom}_{\mathcal{K}_{12}}(a, b) & :=\operatorname{Hom}_{\mathcal{K}_{12}}\left(\rho_{12} \rho_{1}(a), \rho_{12} \rho_{1}(b)\right)=\operatorname{Hom}_{\mathcal{L}_{12}}\left(\rho_{12} \rho_{1}(a), \rho_{12} \rho_{1}(b)\right)
\end{aligned}
$$

the groups of homomorphisms in the various localizations, see (4). More generally, we tend to drop the mention of the restriction functors when it improves readability.

Definition 2.9 Again, as in Definitions 1.10, for objects $a, b \in \mathcal{K}$ and for $i \in\{1,2,12\}$, we refer to a morphism in $\operatorname{Hom}_{\mathcal{K}_{i}}(a, b)$ as a morphism between $a$ and $b$ in $\mathcal{K}_{i}$. By construction of Verdier localizations, any morphism $a \rightarrow b$ in $\mathcal{K}_{i}$ is the equivalence class of a (left) fraction $f s^{-1}=\left(a \longleftarrow^{s} x \stackrel{f}{\longrightarrow} b\right)$ in $\mathcal{K}$, where $s: x \rightarrow a$ is a $\mathcal{K}_{i}$-isomorphism and $f: x \rightarrow b$ is any morphism in $\mathcal{K}$. The equivalence relation on such fractions is given by amplification $f s^{-1}=(f t)(s t)^{-1}$ for any $\mathcal{K}_{i}$-isomorphism $t: y \rightarrow x$.

LEMMA 2.10 In a formal Mayer-Vietoris situation (4), every $\mathcal{K}_{12}$-isomorphism $s: a \rightarrow b$ can be factored as $s=s_{1} \circ s_{2}$ where $s_{i}$ is a $\mathcal{K}_{i}$-isomorphism for $i=1,2$.

Proof. By hypothesis we have that cone $(s) \in \mathcal{J}_{12}=\mathcal{J}_{1} \oplus \mathcal{J}_{2}$. Thus cone $(s)$ may be written as cone $(s) \simeq c_{1} \oplus c_{2}$ where $c_{i} \in \mathcal{K}_{i}$. Now use Lemma 1.13 which tells that $s=\alpha_{0} \delta_{0}$ and that cone $\left(\alpha_{0}\right) \simeq$ $c_{1}$ and cone $\left(\delta_{0}\right) \simeq c_{2}$.

REMARK 2.11 One can actually prove that the above factorization is essentially unique but we shall not use this fact below.

We now give two useful lemmas about weakly bicartesian squares (Definition 1.14).

LEMMA 2.12 Consider a weakly bicartesian square in $\mathcal{K}$ :

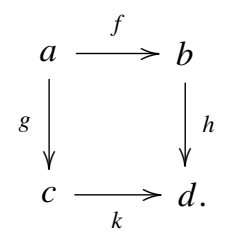

Then $f$ is a $\mathcal{K}_{i}$-isomorphism if and only if $k$ is.

Proof. There exists a distinguished triangle $T^{-1} d \longrightarrow a \stackrel{\left(\begin{array}{l}f \\ g\end{array}\right)}{\longrightarrow} b \oplus c \stackrel{(-h k)}{\longrightarrow} d$. By Lemma 1.13 , cone $(f) \simeq$ cone $(k)$ and the result follows. 
LEMMA 2.13 In a formal Mayer-Vietoris situation (4), consider a commutative square in $\mathcal{K}$ :

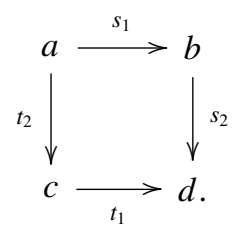

Assume that $s_{i}$ and $t_{i}$ are $\mathcal{K}_{i}$-isomorphisms for $i=1,2$. Then the square is weakly bicartesian.

Proof. Consider the weak push-out $\left(e, u_{1}, u_{2}\right)$ of $s_{1}$ and $t_{2}$ and some morphism $v: e \rightarrow d$ induced by weak push-out of $s_{2}$ and $t_{1}$ :

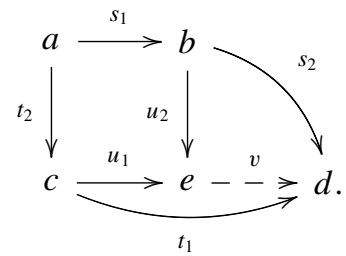

By Lemma 2.12, $u_{i}$ is a $\mathcal{K}_{i}$-isomorphism for $i=1,2$. By 2-out-of-3, $v$ is both a $\mathcal{K}_{1^{-}}$and a $\mathcal{K}_{2}$-isomorphism, hence an isomorphism (see Remark 2.7).

\section{Mayer-Vietoris long exact sequence}

Construction 3.1 Consider a formal Mayer-Vietoris situation (Definition 2.1) and two objects $a, b \in \mathcal{K}$. We define a homomorphism

$$
\begin{aligned}
\partial: \operatorname{Hom}_{\mathcal{K}_{12}}(a, b) & \longrightarrow \operatorname{Hom}_{\mathcal{K}}(a, T(b)) \\
g & \longmapsto \partial(g)
\end{aligned}
$$

as follows. Let $f s^{-1}=(a \stackrel{s}{\longleftarrow} x \stackrel{f}{\longrightarrow} b)$ be a fraction representing a morphism $g \in$ $\operatorname{Hom}_{\mathcal{K}_{12}}(a, b)$. The cone of the $\mathcal{K}_{12}$-isomorphism $s$ belongs to $\mathcal{J}_{12}=\mathcal{J}_{1} \oplus \mathcal{J}_{2}$. So, we can chose a distinguished triangle

$$
\left.x \stackrel{s}{\longrightarrow} a \stackrel{\left(\begin{array}{l}
\alpha \\
\beta
\end{array}\right)}{\longrightarrow} c_{1} \oplus c_{2} \stackrel{(\gamma}{\longrightarrow} T x\right)
$$

where $c_{i} \in \mathcal{J}_{i}$ for $i=1,2$. Note that $\gamma \alpha+\delta \beta=0$, that is, $\gamma \alpha=-\delta \beta$. Define now

$$
\partial(g):=T(f) \circ \gamma \alpha=-T(f) \circ \delta \beta .
$$

This is a morphism in $\operatorname{Hom}_{\mathcal{K}}(a, T(b))$, independent of the choices, see Theorem 3.5. 
REMARK 3.2 Since $T$ is an equivalence, the above construction also induces:

$$
\operatorname{Hom}_{\mathcal{K}_{12}}(T(a), b) \stackrel{\partial}{\longrightarrow} \operatorname{Hom}_{\mathcal{K}}(T(a), T(b)) \stackrel{T^{-1}}{\cong} \operatorname{Hom}_{\mathcal{K}}(a, b)
$$

and we also denote this homomorphism by $\partial$, since no confusion should follow. Explicitly, for a morphism $g=(T(a) \stackrel{s}{\longleftarrow} x \stackrel{f}{\longrightarrow} b)$ in $\operatorname{Hom}_{\mathcal{K}_{12}}(T(a), b)$, choose any distinguished triangle $a \stackrel{\left(\begin{array}{l}\alpha \\ \beta\end{array}\right)}{\longrightarrow} c_{1} \oplus c_{2} \stackrel{(\gamma \delta)}{\longrightarrow} x \stackrel{s}{\longrightarrow} T(a)$ with $c_{i} \in \mathcal{J}_{i}$ for $i=1,2$; then we have $\partial(g)=f \circ \gamma \alpha=$ $-f \circ \delta \beta \in \operatorname{Hom}_{\mathcal{K}}(a, b)$.

REMARK 3.3 Note that the above definition of $\partial$ is asymmetric in the subcategories $\mathcal{J}_{1}$ and $\mathcal{J}_{2}$ because of the sign involved in the definition of $\partial(g)$, see (5). In other words, if we switch $\mathcal{J}_{1}$ and $\mathcal{J}_{2}$, we would get the opposite homomorphism: $-\partial$.

REMARK 3.4 One can also define $\partial: \operatorname{Hom}_{\mathcal{K}_{12}}(a, b) \rightarrow \operatorname{Hom}_{\mathcal{K}}(a, T b)$ by means of right fractions instead of left fractions. We leave it to the reader to show, using Lemma 1.13, that these two definitions agree for judicious choices of signs. The original definition (5) of $\partial$ is obviously natural in the object $b$. Naturality in the object $a$ easily follows from this equivalent construction of $\partial$ via right fractions.

THEOREM 3.5 Consider a formal Mayer-Vietoris cover of an idempotent complete triangulated category $\mathcal{K}$ (Definition 2.1) and consider the categories of Diagram (4). Then, for every pair of objects $a, b \in \mathcal{K}$ there is a natural long exact sequence

$$
\begin{aligned}
& \left.\stackrel{\partial}{\rightarrow} \operatorname{Hom}_{\mathcal{K}}(a, b) \stackrel{\left(\begin{array}{c}
\rho_{1} \\
\rho_{2}
\end{array}\right)}{\longrightarrow} \operatorname{Hom}_{\mathcal{K}_{1}}(a, b) \oplus \operatorname{Hom}_{\mathcal{K}_{2}}(a, b) \stackrel{\left(-\rho_{12} \rho_{21}\right.}{\longrightarrow}\right) \\
& \stackrel{\partial}{\longrightarrow} \operatorname{Hom}_{\mathcal{K}}(a, T b) \rightarrow \cdots,
\end{aligned}
$$

where the connecting homomorphism $\partial$ is defined as in Construction 3.1.

Proof. First, we have to check that the definition of $\partial\left(f s^{-1}\right)$ given in 3.1 does not depend on the choice of the objects $c_{i} \in \mathcal{J}_{i}$ such that cone $(s) \simeq c_{1} \oplus c_{2}$. This is easy, for other $c_{i}$ must be isomorphic to the chosen ones: $c_{1} \oplus c_{2} \simeq c_{1}^{\prime} \oplus c_{2}^{\prime}$ and $c_{i} \in \mathcal{J}_{i}$ forces $c_{i} \simeq c_{i}^{\prime}$ for $i=1$, , by the assumption $\operatorname{Hom}_{\mathcal{K}}\left(c_{i}, c_{j}\right)=0$ for $i \neq j$. The isomorphism $c_{1} \simeq c_{1}^{\prime}$ disappears in the composition $\gamma \circ \alpha$ and a fortiori in $\partial\left(f s^{-1}\right)$.

Then, we have to check that $\partial\left(f s^{-1}\right)$ only depends on the class of the fraction $f s^{-1}$. To see this, let $t: y \rightarrow x$ be a $\mathcal{K}_{12}$-isomorphism and let $(f t)(s t)^{-1}=(a \stackrel{s t}{\longleftarrow} y \stackrel{f t}{\longrightarrow} b)$ be the amplified 
fraction. We have to show that $\partial\left((f t)(s t)^{-1}\right)=\partial\left(f s^{-1}\right)$. The morphism $s t$ fits in a distinguished triangle

$$
y \stackrel{s t}{\longrightarrow} a \stackrel{\left(\begin{array}{c}
\alpha^{\prime} \\
\beta^{\prime}
\end{array}\right)}{\longrightarrow} d_{1} \oplus d_{2} \stackrel{\left(\gamma^{\prime} \delta^{\prime}\right)}{\longrightarrow} T y,
$$

where $d_{i} \in \mathcal{J}_{i}$. Comparing the triangles for $s$ and for $s t$ yields the diagram

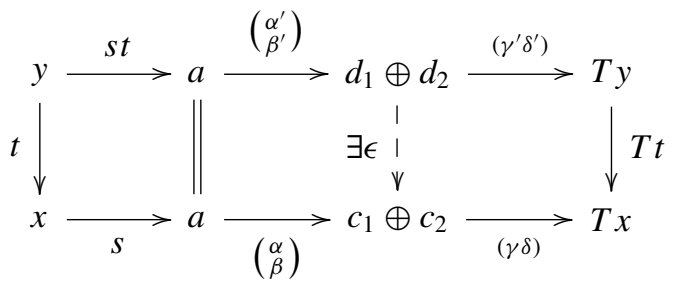

for some morphism $\epsilon$. But since $\operatorname{Hom}_{\mathcal{K}}\left(d_{i}, c_{j}\right)=0$ for $i \neq j$ by the formal Mayer-Vietoris assumption, we have that $\epsilon=\left(\begin{array}{cc}\epsilon_{1} & 0 \\ 0 & \epsilon_{2}\end{array}\right)$. Now compute

$$
\partial\left((f t)(s t)^{-1}\right) \stackrel{\text { def. }}{=} T(f) T(t) \gamma^{\prime} \alpha^{\prime} \stackrel{(6)}{=} T(f) \gamma \epsilon_{1} \alpha^{\prime} \stackrel{(6)}{=} T(f) \gamma \alpha \stackrel{\text { def. }}{=} \partial\left(f s^{-1}\right) .
$$

This proves that $\partial$ is well defined. The fact that $\partial$ does not depend on the amplification of the fraction also shows that in order to prove that $\partial$ is a group homomorphism it suffices to see that $\partial\left((f+g) s^{-1}\right)=\partial\left(f s^{-1}\right)+\partial\left(g s^{-1}\right)$, which is immediate from the definition of $\partial$.

We now prove that the sequence is exact. It is easy to see that all consecutive compositions are zero. (Recall the notation for the restriction functors $\rho_{i}$ for $i \in\{1,2,12,21\}$ from Construction 2.4.) For instance, for $i=1,2$, we have $\rho_{i}\left(\partial\left(f s^{-1}\right)\right)=0$ because $\partial\left(f s^{-1}\right)$ factors via $c_{i} \in \mathcal{J}_{i}$ which becomes zero in $\mathcal{K}_{i}$. To see that $\partial \circ \rho_{12}=0$, we check that $\partial\left(f s^{-1}\right)=0$ if $s$ is a $\mathcal{K}_{1}$-isomorphism. In this case, $c_{2}=0$ and $\partial\left(f s^{-1}\right)$ factors via $c_{2}$. One proves $\partial \circ \rho_{21}=0$ similarly.

Exactness at $\operatorname{Hom}_{\mathcal{K}_{1}}(a, b) \oplus \operatorname{Hom}_{\mathcal{K}_{2}}(a, b)$ : Let $\left(f_{1}, f_{2}\right) \in \operatorname{Hom}_{\mathcal{K}_{1}}(a, b) \oplus \operatorname{Hom}_{\mathcal{K}_{2}}(a, b)$ such that $\rho_{12}\left(f_{1}\right)=\rho_{21}\left(f_{2}\right)$. Write $f_{i}=\left(a \stackrel{s_{i}}{\longleftarrow} x_{i} \stackrel{g_{i}}{\longrightarrow} b\right)$. Then there exist an object $x$ and $\mathcal{K}_{12^{-}}$ isomorphisms $t_{i}: x \rightarrow x_{i}$ such that the diagram

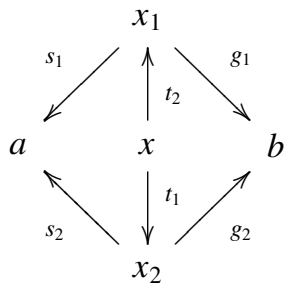

is commutative in $\mathcal{K}$. By Lemma 2.10 we know that every $\mathcal{K}_{12}$-isomorphism factors as a $\mathcal{K}_{1}$ isomorphism followed by a $\mathcal{K}_{2}$-isomorphism (and viceversa) so that we may choose $t_{2}$ to be a $\mathcal{K}_{2}$-isomorphism, up to possibly amplifying the fraction $g_{1} s_{1}^{-1}$ without changing the morphism $f_{1}$ in $\mathcal{K}_{1}$. Similarly, we can assume $t_{1}$ is a $\mathcal{K}_{1}$-isomorphism. By Lemma 2.13 , the left 'square' of (7) is 
weakly bicartesian. Therefore (weak push-out), $g_{1}$ and $g_{2}$ induce a morphism $f \in \operatorname{Hom}_{\mathcal{K}}(a, b)$ such that $f \circ s_{i}=g_{i}$ for $i=1,2$. Hence $f=g_{i} s_{i}^{-1}=f_{i}$ in $\mathcal{K}_{i}$ as wanted.

Exactness at $\operatorname{Hom}_{\mathcal{K}}(a, b)$ : Let $f \in \operatorname{Hom}_{\mathcal{K}}(a, b)$ be such that $\rho_{i}(f)=0$ in $\mathcal{L}_{i}=\mathcal{K} / \mathcal{J}_{i}$ for $i=1,2$. This means that $f$ factors through objects $c_{i} \in \mathcal{J}_{i}$ as follows:

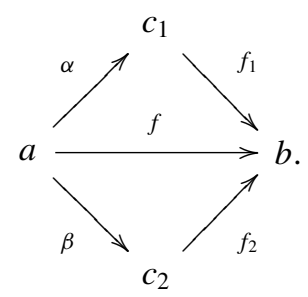

Take now $x$ the weak push-out of $\alpha$ and $\beta$. By construction of the weak push-out (Definition 1.14), we have a distinguished triangle as in the first row of the diagram below. Since $\left(f_{1}-f_{2}\right) \cdot\left(\begin{array}{l}\alpha \\ \beta\end{array}\right)=$ $f-f=0$, there exists a morphism $h: x \rightarrow b$ as follows:

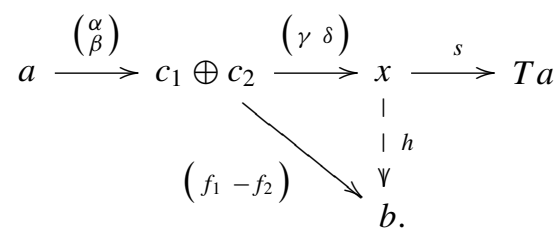

We obtain a morphism $h s^{-1}=(T a \stackrel{s}{\longleftarrow} x \stackrel{h}{\longrightarrow} b) \in \operatorname{Hom}_{\mathcal{K}_{12}}(T a, b)$. By Construction 3.1 and Remark 3.2, we have $\partial\left(h s^{-1}\right)=h \gamma \alpha=f_{1} \alpha=f$.

Exactness at $\operatorname{Hom}_{\mathcal{K}_{12}}(a, b)$ : Let $f s^{-1}=(a \stackrel{s}{\longleftarrow} x \stackrel{f}{\longrightarrow} b)$ be a morphism in $\mathcal{K}_{12}$ such that $\partial\left(f s^{-1}\right)=0$. As in Construction 3.1, choose a distinguished triangle

$$
x \stackrel{s}{\longrightarrow} a \stackrel{\left(\begin{array}{l}
\alpha \\
\beta
\end{array}\right)}{\longrightarrow} c_{1} \oplus c_{2} \stackrel{\left(\begin{array}{ll}
\gamma & \delta
\end{array}\right)}{\longrightarrow} T x,
$$

with $c_{i} \in \mathcal{J}_{i}$. The assumption $\partial\left(f s^{-1}\right)=0$ reads $T(f) \gamma \alpha=0$. Now apply Lemma 1.13 to the above triangle to produce objects $d, e \in \mathcal{K}$ and morphisms $\alpha_{0}, \beta_{0}, \gamma_{0}$ and $\delta_{0}$ satisfying all the conclusions of Lemma 1.13, which the reader is encouraged to have at hand. Recall in particular that $\alpha_{2}=T\left(\delta_{0}\right) \gamma$.

Claim: There exists a distinguished triangle of the form

$$
b \stackrel{\gamma \alpha}{\longrightarrow} T a \stackrel{\left(\begin{array}{c}
T \delta_{0} \\
-T \gamma_{0}
\end{array}\right)}{\longrightarrow} T d \oplus T e \longrightarrow T b .
$$


Indeed, the composition $\alpha_{2} \gamma_{1}=T\left(\delta_{0}\right) \gamma \gamma_{1}=0$ yields an octahedron (the triangles over $\gamma_{1}$ and $\alpha_{2}$ are taken from the conclusions of Lemma 1.13):

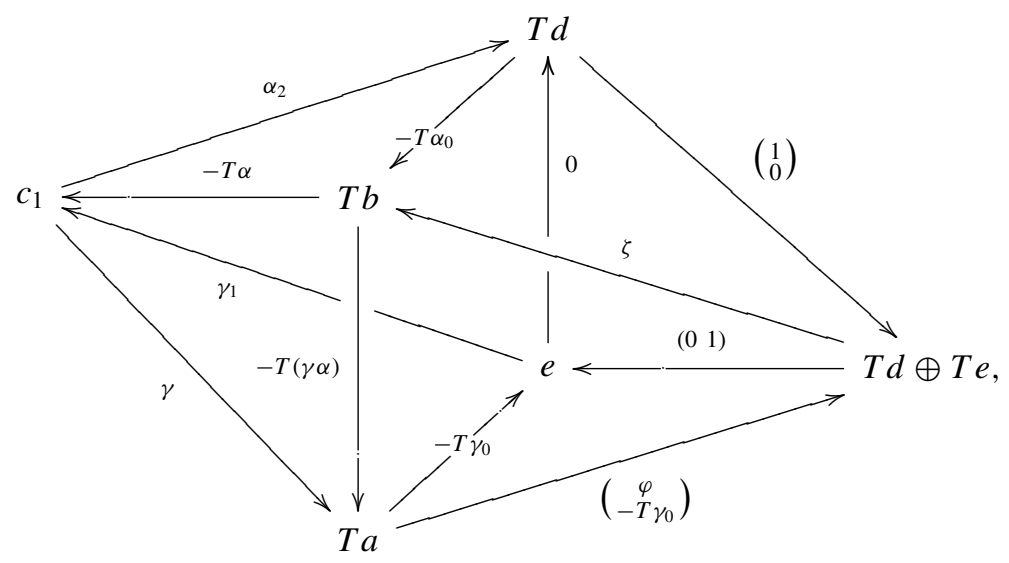

for some morphisms $\varphi$ and $\zeta$. Note in particular the distinguished triangle

$$
b \stackrel{\gamma \alpha}{\longrightarrow} T a \stackrel{\left(\begin{array}{c}
\varphi \\
-T \gamma_{0}
\end{array}\right)}{\longrightarrow} T d \oplus T e \stackrel{\zeta}{\longrightarrow} T b .
$$

To obtain Triangle (8), we still need to 'replace' $\varphi$ by $T \delta_{0}$. Observe that we have $\left(T\left(\delta_{0}\right)-\varphi\right) \circ \gamma=$ $\alpha_{2}-\alpha_{2}=0$. By the distinguished triangle over $\gamma$, there exists a morphism $h: T e \rightarrow T d$ such that $T \delta_{0}-\varphi=h T \gamma_{0}$. Using this equality, we get an isomorphism of triangles

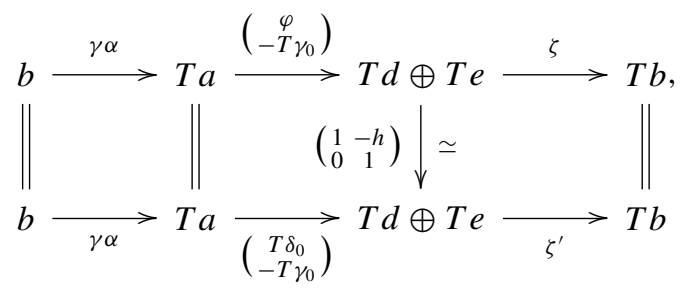

for $\zeta^{\prime}:=\zeta \cdot\left(\begin{array}{ll}1 & h \\ 0 & 1\end{array}\right)$. So, the lower triangle is distinguished and fulfills the Claim.

Using Triangle (8) and the assumption $T(f) \circ \gamma \alpha=\partial\left(f s^{-1}\right)=0$ yields a factorization of $T f$ as follows:

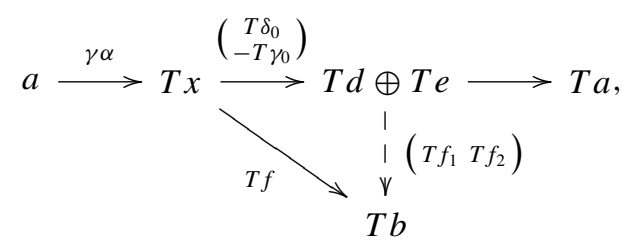

for some morphisms $f_{1}: d \rightarrow b$ and $f_{2}: e \rightarrow b$. This reads $f=f_{1} \delta_{0}-f_{2} \gamma_{0}$. Using the triangles of Lemma 1.13 , it is easy to see that $\alpha_{0}, \gamma_{0}$ are $\mathcal{K}_{1}$-isomorphisms and that $\beta_{0}$ and $\delta_{0}$ are 
$\mathcal{K}_{2}$-isomorphisms. Consider now the morphisms $f_{1} \alpha_{0}^{-1}=\left(a \stackrel{\alpha_{0}}{\longleftarrow} d \stackrel{f_{1}}{\longrightarrow} b\right)$ and $f_{2} \beta_{0}^{-1}=$ $\left(a \stackrel{\beta_{0}}{\longleftarrow} e \stackrel{f_{2}}{\longrightarrow} b\right)$ in $\mathcal{K}_{1}$ and $\mathcal{K}_{2}$, respectively. When restricted to $\mathcal{K}_{12}$ they clearly satisfy $f_{1} \alpha_{0}^{-1}-f_{2} \beta_{0}^{-1}=\left(f_{1} \delta_{0}\right)\left(\alpha_{0} \delta_{0}\right)^{-1}-\left(f_{2} \gamma_{0}\right)\left(\beta_{0} \gamma_{0}\right)^{-1}=f s^{-1}$. The last equation uses the relation $s=\alpha_{0} \delta_{0}=\beta_{0} \gamma_{0}$ from Lemma 1.13 and the above relation $f=f_{1} \delta_{0}-f_{2} \gamma_{0}$.

\section{Gluing of objects}

Definition 4.1 Consider a formal Mayer-Vietoris cover of an idempotent complete triangulated category $\mathcal{K}$, by means of two subcategories $\mathcal{J}_{1}$ and $\mathcal{J}_{2}$ (see Definition 2.1). Recall Diagram (4). Consider two objects $a_{1} \in \mathcal{K}_{1}$ and $a_{2} \in \mathcal{K}_{2}$ and an isomorphism $\sigma: a_{1} \stackrel{\sim}{\rightarrow} a_{2}$ in $\mathcal{K}_{12}$. A gluing of the objects $a_{i}$ along the isomorphism $\sigma$ is an object $a \in \mathcal{K}$ and two isomorphisms $f_{i}: a \stackrel{\sim}{\rightarrow} a_{i}$ in $\mathcal{K}_{i}$ for $i=1,2$ such that $\sigma f_{1}=f_{2}$ in $\mathcal{K}_{12}$. An isomorphism of gluings $\alpha:\left(a, f_{1}, f_{2}\right) \stackrel{\sim}{\rightarrow}\left(a^{\prime}, f_{1}^{\prime}, f_{2}^{\prime}\right)$ is an isomorphism $\alpha: a \stackrel{\sim}{\rightarrow} a^{\prime}$ in $\mathcal{K}$ such that $f_{i}^{\prime} \alpha=f_{i}$ in $\mathcal{K}_{i}$ for all $i=1,2$.

We first prove the gluing of objects without idempotent completions.

LEMMA 4.2 In a formal Mayer-Vietoris situation (Definition 2.1)

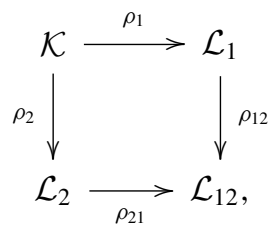

two objects $a_{1} \in \mathcal{L}_{1}, a_{2} \in \mathcal{L}_{2}$ with an isomorphism $\sigma: \rho_{12}\left(a_{1}\right) \stackrel{\sim}{\rightarrow} \rho_{21}\left(a_{2}\right)$ in $\mathcal{L}_{12}$ always admit a gluing (Definition 4.1).

Proof. The isomorphism $\sigma$ can be represented by a fraction $a_{1} \stackrel{s}{\stackrel{s}{\longrightarrow}} \stackrel{t}{\longrightarrow} a_{2}$ where $s, t$ both are $\mathcal{K}_{12}$-isomorphisms. By Lemma 2.10, $s$ and $t$ factor as $s=s_{1} s_{2}$ and $t=t_{2} t_{1}$ where $s_{i}, t_{i}$ are $\mathcal{K}_{i^{-}}$ isomorphisms, see the upper part of Diagram (9). Now complete this diagram by taking the weak push-out of $s_{2}$ and $t_{1}$ :

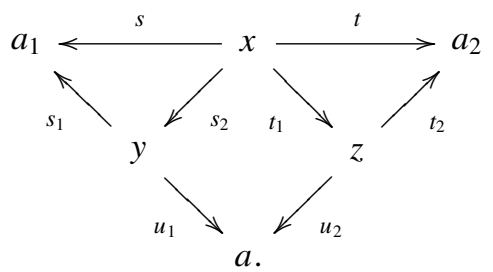

Applying Lemma 2.12, $u_{i}$ is a $\mathcal{K}_{i}$-isomorphism. The object $a$ is then isomorphic to $a_{i}$ in $\mathcal{K}_{i}$ via $f_{1}:=s_{1} \circ u_{1}^{-1}$ and $f_{2}:=t_{2} \circ u_{2}^{-1}$, respectively; the relation $\sigma f_{1}=f_{2}$ is satisfied in $\mathcal{L}_{12}$ because of the commutativity of (9). 
THEOREM 4.3 Assume given a formal Mayer-Vietoris cover of an idempotent complete triangulated category $\mathcal{K}$ (Definition 2.1) and consider the categories constructed in 2.4. Then, given two objects $a_{i} \in \mathcal{K}_{i}$ for $i=1,2$ and an isomorphism $\sigma: \rho_{12}\left(a_{1}\right) \stackrel{\sim}{\rightarrow} \rho_{21}\left(a_{2}\right)$ in $\mathcal{K}_{12}$, there exists a gluing (Definition 4.1). This gluing is unique up to (possibly non-unique) isomorphism of gluings.

Proof. Since $\mathcal{L}_{i}$ is cofinal in $\mathcal{K}_{i}$, we have $a_{i} \oplus T\left(a_{i}\right) \in \mathcal{L}_{i}$ for $i=1,2$ (see [4]). Obviously $\sigma \oplus T \sigma$ gives an isomorphism $\rho_{12}\left(a_{1} \oplus T a_{1}\right) \stackrel{\sim}{\rightarrow} \rho_{21}\left(a_{2} \oplus T a_{2}\right)$ in $\mathcal{K}_{12}$ hence in $\mathcal{L}_{12}$ since $\mathcal{L}_{12} \rightarrow \widetilde{\mathcal{L}_{12}}=\mathcal{K}_{12}$ is fully faithful. By Lemma 4.2, there exists an object $b \in \mathcal{K}$ and isomorphisms $f_{i}: \rho_{i}(b) \rightarrow b_{i}$ in $\mathcal{L}_{i}$ such that $(\sigma \oplus T \sigma) \circ \rho_{12}\left(f_{1}\right)=\rho_{21}\left(f_{2}\right)$. Consider now, for each $i=1,2$ the idempotent $\pi_{i}$ : $\rho_{i}(b) \rightarrow \rho_{i}(b)$ in $\mathcal{L}_{i}$ defined by:

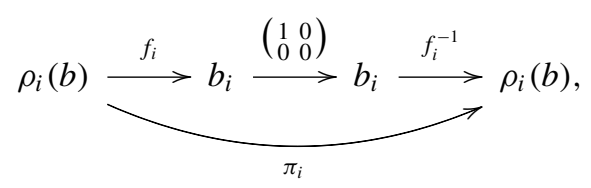

where $\left(\begin{array}{ll}1 & 0 \\ 0 & 0\end{array}\right)$ on $b_{i}=a_{i} \oplus T\left(a_{i}\right)$ is the projection on $a_{i}$. Now, since the diagram

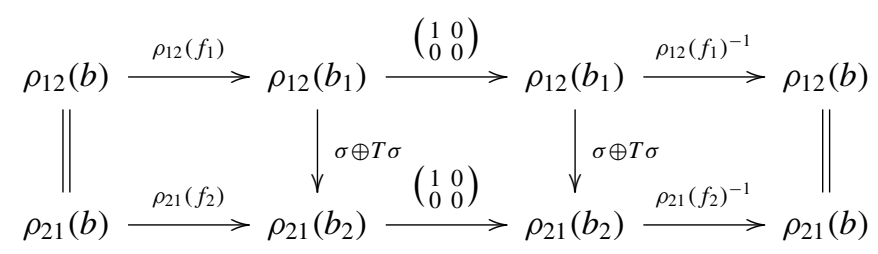

is commutative in $\mathcal{L}_{12}$, we have that $\rho_{12}\left(\pi_{1}\right)=\rho_{21}\left(\pi_{2}\right)$. We can now apply formal Mayer-Vietoris for morphisms (Theorem 3.5) to $\pi_{1}$ and $\pi_{2}$ to show that there exists an endomorphism $\pi: b \rightarrow b$ such that $\rho_{i}(\pi)=\pi_{i}$ for $i=1,2$. We would like $\pi$ to be an idempotent, like its restrictions $\rho_{i}(\pi)$ to $\mathcal{L}_{i}$ for $i=1,2$. Consider $h=\pi^{2}-\pi$. Since $h=0$ in $\mathcal{L}_{i}$ it factors in $\mathcal{K}$ through objects $c_{i} \in \mathcal{J}_{i}$ as follows:

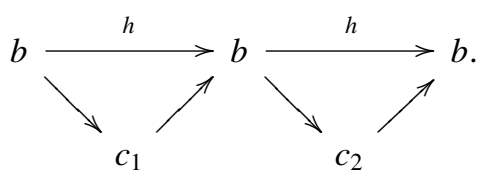

Since $\operatorname{Hom}_{\mathcal{K}}\left(c_{1}, c_{2}\right)=0$ by the formal Mayer-Vietoris assumption, we have $h^{2}=0$. Then, by a standard trick, $p:=\pi+h-2 \pi h$ satisfies $p^{2}=p$ and still has the property $\rho_{i}(p)=\pi_{i}$ since $\rho_{i}(h)=$ 0 . So $p: b \rightarrow b$ is an idempotent lifting $\pi_{i}$ for $i=1,2$. Now, our category $\mathcal{K}$ is idempotent complete by hypothesis, so $b$ splits as $b \simeq \operatorname{Im}(p) \oplus \operatorname{Ker}(p)$. Setting $a=\operatorname{Im}(p)$ gives the desired object with the property $a \simeq a_{i}$ in $\mathcal{K}_{i}$. Further details are left to the reader.

For uniqueness, suppose that $\left(a, f_{1}, f_{2}\right)$ and $\left(a^{\prime}, f_{1}^{\prime}, f_{2}^{\prime}\right)$ are two gluings. By formal Mayer-Vietoris for morphisms (Theorem 3.5) the morphisms $f_{1}^{-1} \circ f_{1}^{\prime}$ and $f_{2}^{-1} \circ f_{2}^{\prime}$ glue into a morphism $a^{\prime} \rightarrow a$ which must be an isomorphism (Remark 2.7). 


\section{Mayer-Vietoris in tensor triangular geometry}

DEFINITION 5.1 Let $\mathcal{K}$ be an idempotent complete strongly closed tensor triangulated category (see section 1). We say that we are in a Mayer-Vietoris situation if the spectrum of $\mathcal{K}$ is covered by two quasi-compact open subsets $\operatorname{Spc}(\mathcal{K})=U_{1} \cup U_{2}$. We shall denote by $Z_{i}=\operatorname{Spc}(\mathcal{K}) \backslash U_{i}$ the closed complements for $i=1,2$.

We now have the key to the results of the previous sections:

THEOREM 5.2 In a Mayer-Vietoris situation as above, the thick subcategories $\mathcal{J}_{1}:=\mathcal{K}_{Z_{1}}$ and $\mathcal{J}_{2}:=$ $\mathcal{K}_{Z_{2}}$ define a formal Mayer-Vietoris cover of $\mathcal{K}$ (Definition 2.1) and $\mathcal{J}_{12}=\mathcal{J}_{1} \oplus \mathcal{J}_{2}$ coincides with $\mathcal{K}_{Z_{1} \cup Z_{2}}$.

Proof. This is Proposition 1.6 and Theorem 1.8.

REMARK 5.3 We do not really need to have $U_{1}$ and $U_{2}$ open and it would be enough to assume that they are arbitrary intersections of quasi-compact open subsets. Indeed, the key result taken from [2], Theorem 1.8, holds in this generality. Therefore, everything below holds in similar generality. We stick to open pieces because this is closer to the reader's understanding of a Mayer-Vietoris framework.

We can now apply the notions and results of section 2, 3 and 4.

DEFINITION 5.4 Recall the important Definitions 1.10. With the simplified notation $\rho_{i}=\rho_{U_{i}}$ and $\rho_{i j}=\rho_{U_{i} \cap U_{j}, U_{i}}$ for the restriction functors, we have the following commutative diagram

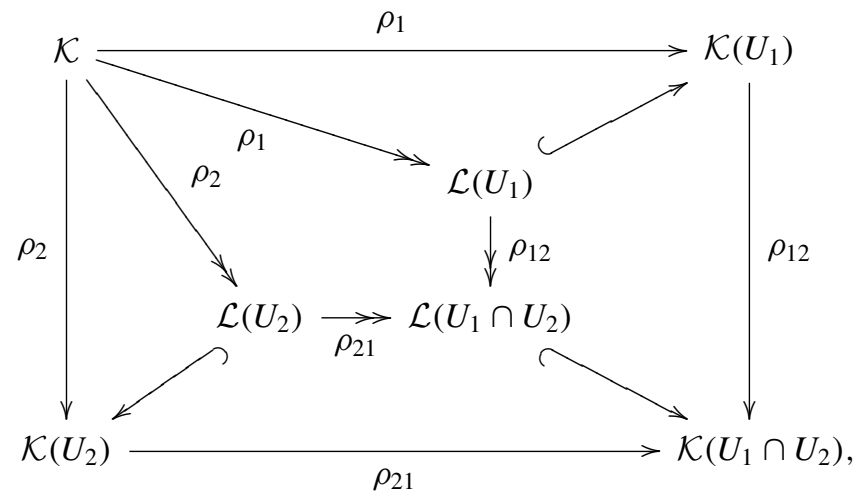

which is exactly Diagram (4) here. Recall that $\rightarrow$ stands for a Verdier localization and $\hookrightarrow$ for a fully faithful cofinal embedding.

Rephrasing Definition 2.6 yields:

Definition 5.5 Let $U \subset \operatorname{Spc}(\mathcal{K})$ be a (quasi-compact) open with closed complement $Z$. A morphism $s: a \rightarrow b$ in $\mathcal{K}$ is called a $U$-isomorphism if it is an isomorphism in $\mathcal{L}(U)$, or equivalently in $\mathcal{K}(U)$. This is also equivalent to saying that cone $(s)$ belongs to $\mathcal{K}_{Z}$ which also reads $\operatorname{supp}(\operatorname{cone}(s)) \cap U=\varnothing$. 
COROLlaRY 5.6 In a Mayer-Vietoris situation, suppose that $s: a \rightarrow b$ is a $U_{1} \cap U_{2}$-isomorphism. Then $s$ may be factored as $s=s_{1} s_{2}$ where $s_{i}$ is a $U_{i}$-isomorphism.

Proof. Lemma 2.10 (and Theorem 5.2).

COROLLARY 5.7 In a Mayer-Vietoris situation, consider a commutative diagram:

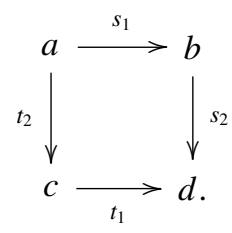

If $s_{i}$ and $t_{i}$ are $U_{i}$-isomorphisms for $i=1,2$, the square is weakly bicartesian.

Proof. Lemma 2.13 (and Theorem 5.2).

COROLlaRy 5.8 (Mayer-Vietoris for morphisms) In a Mayer-Vietoris situation (Definition 5.1), for each pair of objects $a, b \in \mathcal{K}$, the homomorphisms $\partial$ of Construction 3.1 and Remark 3.2 fit in a natural long exact sequence

$$
\cdots \stackrel{\partial}{\rightarrow} \operatorname{Hom}_{\mathcal{K}}(a, b) \rightarrow \operatorname{Hom}_{\mathcal{K}\left(U_{1}\right)}(a, b) \oplus \operatorname{Hom}_{\mathcal{K}\left(U_{2}\right)}(a, b) \rightarrow \operatorname{Hom}_{\mathcal{K}\left(U_{1} \cap U_{2}\right)}(a, b) \stackrel{\partial}{\rightarrow} \cdots,
$$

where the other homomorphisms are the restrictions and differences of restrictions.

Proof. Apply Theorem 3.5 (and Theorem 5.2). Of course, Construction 3.1 should be applied to $\mathcal{J}_{1}=\mathcal{K}_{Z_{1}}$ and $\mathcal{J}_{2}=\mathcal{K}_{Z_{2}}$.

Let us now discuss the gluing of objects. It is convenient to fix the following standard terminology, generalizing Definition 4.1.

DEFINITION 5.9 Let $\operatorname{Spc}(\mathcal{K})=U_{1} \cup \cdots \cup U_{n}$ be a cover by quasi-compact open subsets. Consider objects $a_{i} \in \mathcal{K}\left(U_{i}\right)$ and isomorphisms $\sigma_{j i}: a_{i} \stackrel{\sim}{\rightarrow} a_{j}$ in $\mathcal{K}\left(U_{i} \cap U_{j}\right)$ such that $\sigma_{k i}=\sigma_{k j} \sigma_{j i}$ in $\mathcal{K}\left(U_{i} \cap\right.$ $\left.U_{j} \cap U_{k}\right)$ for $1 \leq i, j, k \leq n$. A gluing of the objects $a_{i}$ along the isomorphisms $\sigma_{i j}$ is an object $a \in \mathcal{K}$ and $n$ isomorphisms $f_{i}: a \stackrel{\sim}{\rightarrow} a_{i}$ in $\mathcal{K}\left(U_{i}\right)$ such that $\sigma_{j i} f_{i}=f_{j}$ in $\mathcal{K}\left(U_{i} \cap U_{j}\right)$ for all $1 \leq i, j \leq n$. An isomorphism of gluings $f:\left(a, f_{1}, \ldots, f_{n}\right) \stackrel{\sim}{\rightarrow}\left(a^{\prime}, f_{1}^{\prime}, \ldots, f_{n}^{\prime}\right)$ is an isomorphism $f: a \stackrel{\sim}{\rightarrow} a^{\prime}$ in $\mathcal{K}$ such that $f_{i}^{\prime} f=f_{i}$ in $\mathcal{K}\left(U_{i}\right)$ for all $i=1, \ldots, n$. (As before, we temporarily dropped the restriction functors for readability.) 
COROLlary 5.10 (Gluing of two objects) In a Mayer-Vietoris situation (Definition 5.1)

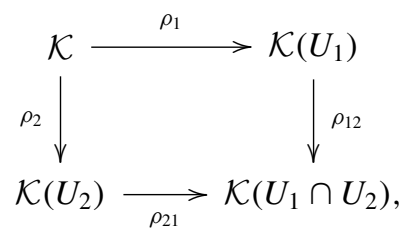

given two objects $a_{i} \in \mathcal{K}\left(U_{i}\right)$ for $i=1,2$ and an isomorphism $\sigma: \rho_{12}\left(a_{1}\right) \stackrel{\sim}{\rightarrow} \rho_{21}\left(a_{2}\right)$ in $\mathcal{K}\left(U_{1} \cap U_{2}\right)$, there exists a gluing (Definition 5.9), which is unique up to (possibly non-unique) isomorphism.

Proof. Apply Theorem 4.3 (and Theorem 5.2).

COROLLARY 5.11 (Gluing of three objects) Let $\operatorname{Spc}(\mathcal{K})=U_{1} \cup U_{2} \cup U_{3}$ be a cover by quasi-compact open subsets. Consider three objects $a_{i} \in \mathcal{K}\left(U_{i}\right)$ for $i=1,2,3$ and three isomorphisms $\sigma_{i j}: a_{j} \stackrel{\sim}{\rightarrow} a_{i}$ in $\mathcal{K}\left(U_{i} \cap U_{j}\right)$ for $1 \leq i<j \leq 3$ satisfying the cocycle relation $\sigma_{12} \circ \sigma_{23}=\sigma_{13}$ in $\mathcal{K}\left(U_{1} \cap U_{2} \cap U_{3}\right)$. Then they admit a gluing.

Proof. Note that $V:=\operatorname{Spc}\left(\mathcal{K}\left(U_{1} \cup U_{2}\right)\right)=U_{1} \cup U_{2}$ by Proposition 1.11. Using gluing of two objects (Corollary 5.10), we can glue $a_{1}$ and $a_{2}$ into an object $b \in \mathcal{K}(V)$. Using Mayer-Vietoris for morphisms (Corollary 5.8) for the cover of $V \cap U_{3}$ by $U_{1} \cap U_{3}$ and $U_{2} \cap U_{3}$, we can construct a (possibly non-unique) isomorphism between $b$ and $a_{3}$ in $\mathcal{K}\left(V \cap U_{3}\right)$. By gluing of two objects (Corollary 5.10) for the cover of $\operatorname{Spc}(\mathcal{K})$ given by $V$ and $U_{3}$, we can now glue $b$ and $a_{3}$ into an object of $\mathcal{K}$.

REMARK 5.12 As the above proof shows, the problem that arises with three open subsets is that the isomorphism between the objects $b \in \mathcal{K}(V)$ and $a_{3} \in \mathcal{K}\left(U_{3}\right)$ on $V \cap U_{3}$ is not unique. Various choices are parameterized by $\operatorname{Hom}_{\mathcal{K}\left(V \cap U_{3}\right)}\left(T a_{1}, a_{2}\right)$ but we were not able to prove that two such choices yield isomorphic gluings and we tend to believe that this is wrong in general. Nevertheless, here is a case where the gluing works for several open subsets. This applies in particular to vector bundles (concentrated in degree zero) in $\mathcal{K}=\mathrm{D}^{\text {perf }}(X)$ and hence recovers the standard gluing of vector bundles in algebraic geometry, for instance.

THEOREM 5.13 (Connective gluing of several objects) Let $\operatorname{Spc}(\mathcal{K})=U_{1} \cup \cdots \cup U_{n}$ be a cover by quasi-compact open subsets for $n \geq 2$. Consider objects $a_{i} \in \mathcal{K}\left(U_{i}\right)$ and isomorphisms $\sigma_{j i}: \stackrel{\sim}{\rightarrow} a_{j}$ in $\mathcal{K}\left(U_{i} \cap U_{j}\right)$ satisfying the cocycle condition $\sigma_{k j} \sigma_{j i}=\sigma_{k i}$ in $\mathcal{K}\left(U_{i} \cap U_{j} \cap U_{k}\right)$ for $1 \leq i, j, k \leq n$. Assume moreover the following Connectivity Condition: For any $i=2, \ldots, n$ and for any quasicompact open $V \subset U_{i}$, we suppose that:

$$
\operatorname{Hom}_{\mathcal{K}(V)}\left(T a_{i}, a_{i}\right)=0 .
$$

(It suffices to have it for the $V \subset U_{i}$ which are unions of intersections of $U_{1}, \ldots, U_{n}$.) Then there exists a gluing (Definition 5.9), which is unique up to unique isomorphism.

Proof. We prove the result by induction on $n$. Let us first establish the $n=2$ case. By gluing of two objects (Corollary 5.10) we only need to prove the uniqueness of the isomorphism. To see this, 
it suffices to prove that for two gluings $a, a^{\prime} \in \mathcal{K}$, two (iso)morphisms $g, g^{\prime}: a \rightarrow a^{\prime}$ which agree in $\mathcal{K}\left(U_{1}\right)$ and $\mathcal{K}\left(U_{2}\right)$ are equal. By Mayer-Vietoris for morphisms (Corollary 5.8), it suffices to show that $\operatorname{Hom}_{\mathcal{K}\left(U_{1} \cap U_{2}\right)}\left(T a, a^{\prime}\right)=0$ which follows from the Connectivity Condition (11) and from $a \simeq a^{\prime} \simeq a_{2}$ in $\mathcal{K}\left(U_{2}\right)$.

Suppose $n \geq 3$ and the result known for $n-1$. Define $V=U_{1} \cup \ldots \cup U_{n-1}$. Since $V$ is quasicompact, we know by Proportion 1.11 that $\operatorname{Spc}(\mathcal{K}(V))=V$ and we can apply the induction hypothesis to construct a gluing $b \in \mathcal{K}(V)$ with isomorphisms $g_{i}: b \stackrel{\sim}{\rightarrow} a_{i}$ in $\mathcal{K}\left(U_{i}\right)$ for $i=1, \ldots, n-1$, such that $\sigma_{i j} g_{j}=g_{i}$ for all $1 \leq i, j \leq n-1$. Consider the intersection $W:=V \cap U_{n}$, which is covered by $n-1$ quasi-compact subsets $W=\left(U_{1} \cap U_{n}\right) \cup \cdots \cup\left(U_{n-1} \cap U_{n}\right)$. In the category $\mathcal{K}(W)$, we have two objects $b$ and $a_{n}$ (that is, their restrictions, of course) which are isomorphic in $\mathcal{K}\left(U_{i} \cap U_{n}\right)$ for $i=1, \ldots, n-1$ in a compatible way with the $\sigma_{i j}$. By uniqueness of the gluing for $n-1$, there exists a unique isomorphism $\sigma: b \stackrel{\sim}{\rightarrow} a_{n}$ on $V \cap U_{n}$ such that $\sigma_{i n} \sigma=g_{i}$ for $i=1, \ldots, n-1$. By the $n=2$ case, we obtain the wanted gluing $a \in \mathcal{K}$ of $b$ and $a_{n}$, unique up to unique isomorphism. Details are left to the careful reader, who will note that uniqueness of the isomorphism $\sigma$ (at stage $n-1$ ) is essential for uniqueness of the gluing $a$ (at stage $n$ ).

In the above induction, we needed that if the tuple $\left(U_{1}, \ldots, U_{n} ; a_{1}, \ldots, a_{n}\right)$ satisfies the Connectivity Condition (11) for $n$, then:

- the tuple $\left(U_{1}, \ldots, U_{n-1} ; a_{1}, \ldots, a_{n-1}\right)$ satisfies (11) for $n-1$,

- the tuple $\left(U_{1} \cap U_{n}, \ldots, U_{n-1} \cap U_{n} ; a_{1}, \ldots, a_{n-1}\right)$ satisfies (11) for $n-1$,

- the 4-uple $\left(U_{1} \cup \ldots \cup U_{n-1}, U_{n} ; b, a_{n}\right)$ satisfies (11) for $n=2$, for any object $b$. These are easy to check. The last one comes from the assumption $i>1$ in (11).

\section{Picard groups}

The next definition is an elementary fact for line bundles in algebraic geometry and its generalization to (closed) symmetric monoidal category roots back to the French geometer Jacques II de Chabannes (1470-1525). See also [6] and further references therein.

Definition 6.1 An object $a \in \mathcal{K}$ is called invertible if there exists an object $b$ such that $a \otimes b \simeq \mathbf{1}$. By adjunction, see Definition 1.4, an object $a \in \mathcal{K}$ is invertible if and only if the evaluation map $\eta: D a \otimes a \rightarrow \mathbf{1}$ is an isomorphism.

Lemma 6.2 An object a in $\mathcal{K}$ is invertible if and only if it is invertible in $\mathcal{K} / \mathcal{P}$ for all $\mathcal{P} \in \operatorname{Spc}(\mathcal{K})$.

Proof. The evaluation map $\eta: D a \otimes a \rightarrow \mathbf{1}$ is an isomorphism by Proposition 1.7.

Definition 6.3 Here we call Picard group, $\operatorname{Pic}(\mathcal{K})$, the set of isomorphism classes of invertible objects in $\mathcal{K}$. The tensor product $\otimes: \mathcal{K} \times \mathcal{K} \rightarrow \mathcal{K}$ makes $\operatorname{Pic}(\mathcal{K})$ into an abelian group with unit the class of 1 . (The reader should be aware that some authors can call Picard group something different, like sometimes the set of all auto-equivalences of $\mathcal{K}$.)

The following, essentially obvious result is well known. We give a short proof for the convenience of the reader. 
Proposition 6.4 Let $X$ be a scheme and consider $\mathrm{D}^{\text {perf }}(X)$ its derived category of perfect complexes. Then there is a split short exact sequence of abelian groups

$$
0 \rightarrow \operatorname{Pic}(X) \rightarrow \operatorname{Pic}\left(\mathrm{D}^{\text {perf }}(X)\right) \rightarrow C(X ; \mathbb{Z}) \rightarrow 0,
$$

where $C(X ; \mathbb{Z})$ stands for the group of locally constant functions from $X$ to $\mathbb{Z}$.

Proof. We first describe $\operatorname{Pic}\left(\mathrm{D}^{\text {perf }}(X)\right)$, where $X=\operatorname{Spec}(R)$ is the spectrum of a local ring $(R, \mathfrak{m})$. In this case, any object of $\mathrm{D}^{\text {perf }}(R)$ is isomorphic to a so-called minimal complex of the form

$$
C=\cdots \longrightarrow R^{\ell_{i}} \stackrel{d_{i}}{\longrightarrow} R^{\ell_{i-1}} \longrightarrow \cdots,
$$

where, for all $i$, the differential $d_{i}$ is a matrix with coefficients in $\mathfrak{m}$. If $C$ is invertible in $\mathrm{D}^{\text {perf }}(R)$ so is $\bar{C}$, its image under the functor $\mathrm{D}^{\text {perf }}(R) \rightarrow \mathrm{D}^{\text {perf }}(R / \mathfrak{m})$. But all the differentials of $\bar{C}$ are 0 and the relation $C \otimes D \simeq R$, for some complex $D$, hence $\bar{C} \otimes \bar{D} \simeq R / \mathfrak{m}$, shows that the complex $C$ must be $R$ concentrated in some degree, that is, there exists $n_{0}=n_{0}(C ; R)$ such that $\ell_{i}=1$ if $i=n_{0}$ and $\ell_{i}=0$ otherwise.

For a global $X$, the map $\operatorname{Pic}\left(\mathrm{D}^{\text {perf }}(X)\right) \rightarrow C(X ; \mathbb{Z})$ is now easily defined: for an invertible complex $C \in \mathrm{D}^{\text {perf }}(X)$ and for $x \in X$ denote by $C_{x}$ its image in $\mathrm{D}^{\text {perf }}\left(\mathcal{O}_{X, x}\right)$. The function $f_{C}: X \rightarrow \mathbb{Z}$ is then defined by $x \mapsto n_{0}\left(C_{x} ; \mathcal{O}_{X, x}\right)$. The rest of the proof is straightforward: a perfect complex which is locally trivial is quasi-isomorphic to its homology in degree zero, which must be a line bundle.

REMARK 6.5 The result is the same if one works with bigger derived categories instead of $\mathrm{D}^{\text {perf }}(X)$. See for instance Fausk's paper [6]. This might look more general than the above Proposition but one should keep in mind that invertible objects in such big categories are necessarily compact, just by abstract non-sense, see [7, Proposition A.2.8] for instance. So, the above argument already contains most of the relevant algebraic geometry for this problem.

Definition 6.6 Define $\mathbb{G}_{\mathrm{m}}(\mathcal{K})=\operatorname{Hom}_{\mathcal{K}}(\mathbf{1}, \mathbf{1})^{\times}$to be the group of invertible elements of the (commutative) ring $\operatorname{Hom}_{\mathcal{K}}(\mathbf{1}, \mathbf{1})$.

THEOREM 6.7 In a Mayer-Vietoris situation (Definition 5.1), there is an exact sequence of abelian groups

$$
\begin{aligned}
& \cdots \longrightarrow \operatorname{Hom}_{\mathcal{K}\left(U_{1} \cap U_{2}\right)}(T \mathbf{1}, \mathbf{1}) \stackrel{1+\partial}{\longrightarrow} \\
& \stackrel{1+\partial}{\longrightarrow} \mathbb{G}_{\mathrm{m}}(\mathcal{K}) \longrightarrow \mathbb{G}_{\mathrm{m}}\left(\mathcal{K}\left(U_{1}\right)\right) \oplus \mathbb{G}_{\mathrm{m}}\left(\mathcal{K}\left(U_{2}\right)\right) \longrightarrow \mathbb{G}_{\mathrm{m}}\left(\mathcal{K}\left(U_{1} \cap U_{2}\right)\right) \stackrel{\delta}{\longrightarrow} \\
& \stackrel{\delta}{\longrightarrow} \operatorname{Pic}(\mathcal{K}) \longrightarrow \operatorname{Pic}\left(\mathcal{K}\left(U_{1}\right)\right) \oplus \operatorname{Pic}\left(\mathcal{K}\left(U_{2}\right)\right) \longrightarrow \operatorname{Pic}\left(\mathcal{K}\left(U_{1} \cap U_{2}\right)\right) .
\end{aligned}
$$

The homomorphism $\partial$ is as in Construction 3.1 and the unlabelled homomorphisms are the restrictions and the (multiplicative) differences thereof. 
The homomorphism $\delta: \mathbb{G}_{\mathrm{m}}\left(\mathcal{K}\left(U_{1} \cap U_{2}\right)\right) \rightarrow \operatorname{Pic}(\mathcal{K})$ is defined by gluing two copies of $\mathbf{1}$ by means of Corollary 5.10. Explicitly, it can be described as follows: Let $\sigma=t s^{-1}: \quad \mathbf{1} \stackrel{s}{ }^{s} \stackrel{t}{\longrightarrow} \mathbf{1}$ in $\mathbb{G}_{\mathrm{m}}\left(\mathcal{K}\left(U_{1} \cap U_{2}\right)\right)$, where $s$ and $t$ are $U_{1} \cap U_{2}$-isomorphisms; by Corollary 5.6, there exist factorizations $s=s_{1} s_{2}$ and $t=t_{2} t_{1}$ where $s_{i}$ and $t_{i}$ are $U_{i}$-isomorphisms; then $\delta(\sigma)$ is defined as the isomorphism class of the weak push-out $p \in \mathcal{K}$ of $x_{1}$ and $x_{2}$ over $x$

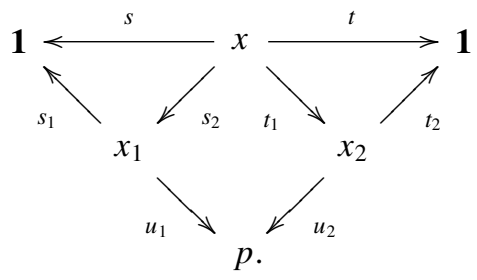

Proof. First note that the homomorphism $1+\partial: \operatorname{Hom}_{\mathcal{K}\left(U_{1} \cap U_{2}\right)}(T \mathbf{1}, \mathbf{1}) \rightarrow \operatorname{Hom}_{\mathcal{K}}(\mathbf{1}, \mathbf{1})$ defined by $g \mapsto 1+\partial(g)$ lands in $\mathbb{G}_{\mathrm{m}}(\mathcal{K})$. Indeed for any $g \in \operatorname{Hom}_{\mathcal{K}\left(U_{1} \cap U_{2}\right)}(T \mathbf{1}, \mathbf{1})$ one has $\partial(g) \circ \partial(g)=0$, since $\partial(g): \mathbf{1} \rightarrow \mathbf{1}$ is zero in $\mathcal{K}\left(U_{i}\right)$ and hence factors via some object of $\mathcal{K}_{Z_{i}}$ for $i=1,2$ and since $\operatorname{Hom}_{\mathcal{K}}\left(\mathcal{K}_{Z_{1}}, \mathcal{K}_{Z_{2}}\right)=0$ by Proposition 1.6. So, $1+\partial(g)$ is invertible with inverse $1-\partial(g)$.

The connecting homomorphism $\delta: \mathbb{G}_{\mathrm{m}}\left(\mathcal{K}\left(U_{1} \cap U_{2}\right)\right) \rightarrow \operatorname{Pic}(\mathcal{K})$ produces an object $p \in \mathcal{K}$, see Diagram (12), which is isomorphic to 1 in $\mathcal{K}\left(U_{1}\right)$ via $s_{1} u_{1}^{-1}$ and in $\mathcal{K}\left(U_{2}\right)$ via $t_{2} u_{2}^{-1}$, in a compatible way with $\sigma$ in $\mathcal{K}\left(U_{1} \cap U_{2}\right)$. The object $p$ is then the gluing of two copies of 1 along the isomorphism $\sigma$ in $\mathcal{K}\left(U_{1} \cap U_{2}\right)$. Such a gluing is unique up to isomorphism by Corollary 5.10 , and this gluing only depends on the map $\sigma$, and not on the various choices $\left(s, t, s_{1}, s_{2}, t_{1}, t_{2}, p\right)$. So, the map $\delta$ is well defined and we now check that it is a group homomorphism. Take $\mathbf{1} \stackrel{s}{\longleftarrow} x \stackrel{t}{\longrightarrow} \mathbf{1}$ and $\mathbf{1} \stackrel{s^{\prime}}{\longleftarrow} x^{\prime} \stackrel{t^{\prime}}{\longrightarrow} \mathbf{1}$ two units in $\mathbb{G}_{\mathrm{m}}\left(\mathcal{K}\left(U_{1} \cap U_{2}\right)\right)$. With the same notations as above, factor these morphisms and perform the corresponding weak push-outs:
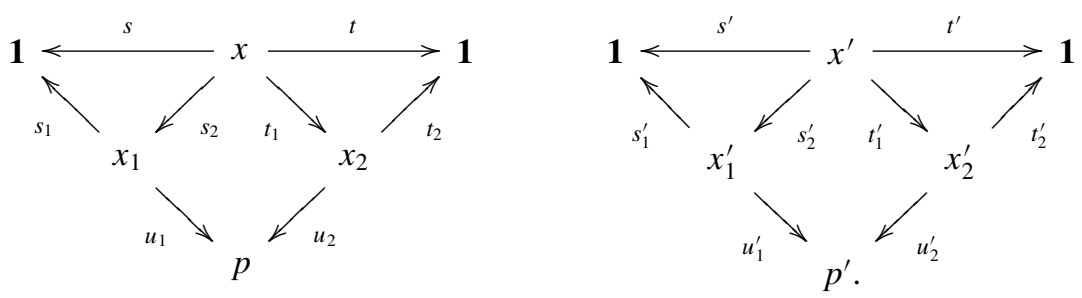
In a symmetric monoidal category, the composition of two endomorphisms of the unit object is equal to their tensor product. Hence we tensor together the two above diagrams to obtain the following one:

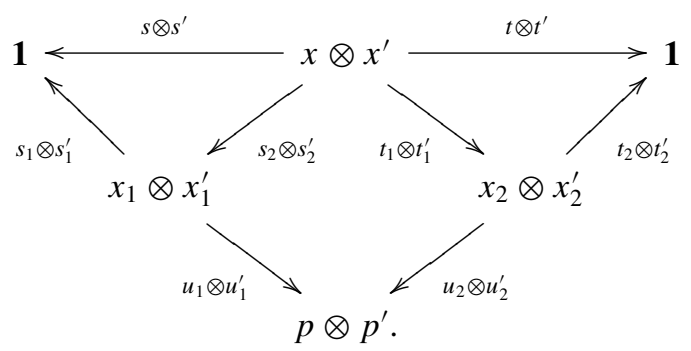

By Corollary 5.7, the above middle square is weakly bicartesian as well. Hence, $p \otimes p^{\prime}=\delta(\sigma \otimes$ $\left.\sigma^{\prime}\right)=\delta\left(\sigma \sigma^{\prime}\right)$. This shows that $\delta$ is an group homomorphism.

(Recall the restriction functors $\rho_{i}$ and $\rho_{i j}$ from Definition 5.4.) It is straightforward from the above definition of $\delta$ that $\rho_{i} \circ \delta=0$ for $i=1,2$. To see that $\delta \circ \rho_{12}=0$, for instance, one can assume that $s_{2}=\mathrm{id}$ and $t_{2}=\mathrm{id}_{\mathbf{1}}$ in (12), in which case $u_{2}$ must also be an isomorphism, that is, $p \simeq x_{2}=\mathbf{1}$. The other compositions are clearly 0 (keeping in mind that 0 means 1 or $\mathbf{1}$ in the multiplicative groups $\mathbb{G}_{\mathrm{m}}$ and Pic). The exactness of the left-hand side of the sequence up to $\mathbb{G}_{\mathrm{m}}(\mathcal{K})$ follows from MayerVietoris for morphisms (Corollary 5.8) applied at $a=b=\mathbf{1}$. It remains to check the exactness of the sequence at four spots.

Exactness at $\mathbb{G}_{\mathrm{m}}\left(\mathcal{K}\left(U_{1}\right)\right) \oplus \mathbb{G}_{\mathrm{m}}\left(\mathcal{K}\left(U_{2}\right)\right)$ : This is again immediate from Mayer-Vietoris for morphisms (Corollary 5.8) recalling that a local isomorphism is an isomorphism (Proposition 1.7).

Exactness at $\mathbb{G}_{\mathrm{m}}\left(\mathcal{K}\left(U_{1} \cap U_{2}\right)\right)$ : Let $\sigma=(\mathbf{1} \stackrel{s}{\longleftarrow} x \stackrel{t}{\longrightarrow} \mathbf{1})$ in $\mathbb{G}_{\mathrm{m}}\left(\mathcal{K}\left(U_{1} \cap U_{2}\right)\right)$ be such that $\delta(\sigma) \simeq \mathbf{1}$ in $\mathcal{K}$. This means that one can find a diagram of the form

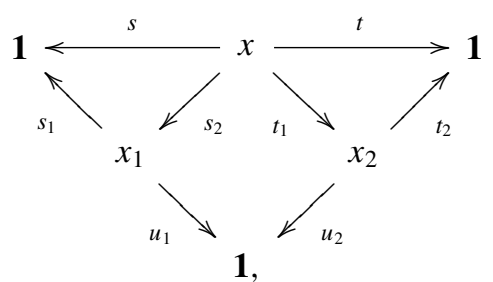

see (12). One then sees two morphisms, namely $\sigma_{1}=u_{1} s_{1}^{-1} \in \mathbb{G}_{\mathrm{m}}\left(\mathcal{K}\left(U_{1}\right)\right)$ and $\sigma_{2}=u_{2} t_{2}^{-1} \in$ $\mathbb{G}_{\mathrm{m}}\left(\mathcal{K}\left(U_{2}\right)\right)$, such that $\sigma_{2}^{-1} \circ \sigma_{1}=\sigma$ in $\mathbb{G}_{\mathrm{m}}\left(\mathcal{K}\left(U_{1} \cap U_{2}\right)\right)$.

Exactness at $\operatorname{Pic}(\mathcal{K})$ : Let $p$ be an invertible object in $\mathcal{K}$ such that $\rho_{i}(p) \simeq \mathbf{1}$ for $i=1,2$. Thus there exist $U_{i}$-isomorphisms $\mathbf{1} \stackrel{s_{i}}{\longleftarrow} y_{i} \stackrel{t_{i}}{\longrightarrow} p$. Performing the weak pull-back of $s_{1}$ and $s_{2}$ one obtains 
the diagram

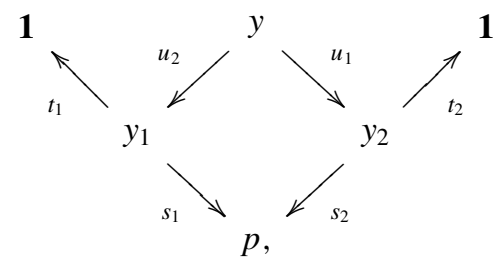

and this defines $\left(\mathbf{1} \stackrel{t_{1} u_{2}}{\longleftarrow} y \stackrel{t_{2} u_{1}}{\longrightarrow} \mathbf{1}\right) \in \mathbb{G}_{\mathrm{m}}\left(\mathcal{K}\left(U_{1} \cap U_{2}\right)\right)$. The image of this morphism under $\delta$ is clearly isomorphic to $p$ by construction, see (12), the middle square in the above diagram being weakly bicartesian.

Exactness at $\operatorname{Pic}\left(\mathcal{K}\left(U_{1}\right)\right) \oplus \operatorname{Pic}\left(\mathcal{K}\left(U_{2}\right)\right)$ : This follows from gluing of two objects (Corollary 5.10) and from invertibility being a local property (see Lemma 6.2).

THEOREM 6.8 Let $\mathcal{K}$ be an idempotent complete strongly closed tensor triangulated category. Suppose that $\operatorname{Hom}_{\mathcal{K}(U)}(T \mathbf{1}, \mathbf{1})=0$ for every quasi-compact open subsets $U \subset \operatorname{Spc}(\mathcal{K})$. Then there exists a unique sheaf $\mathbb{G}_{\mathrm{m}}$ on $\operatorname{Spc}(\mathcal{K})$, such that $\mathbb{G}_{\mathrm{m}}(U)=\mathbb{G}_{\mathrm{m}}(\mathcal{K}(U))$ when $U \subset \operatorname{Spc}(\mathcal{K})$ is quasi-compact open. Moreover, there exists an injective homomorphism from the first Čech cohomology of $\operatorname{Spc}(\mathcal{K})$ with coefficients in $\mathbb{G}_{\mathrm{m}}$ into the Picard group of $\mathcal{K}$

$$
\alpha: \check{\mathrm{H}}^{1}\left(\operatorname{Spc}(\mathcal{K}), \mathbb{G}_{\mathrm{m}}\right) \hookrightarrow \operatorname{Pic}(\mathcal{K})
$$

which sends a $\mathbb{G}_{\mathrm{m}}$-cocycle $\sigma$ to the unique gluing of copies of $\mathbf{1}$ along the isomorphisms over the pairwise intersections given by $\sigma$, as described in Theorem 5.13.

Proof. We first prove by induction on $n$ the following

Claim: Given a cover of a quasi-compact subset $V \subset \operatorname{Spc}(\mathcal{K})$ by $n \geq 2$ quasi-compact open subsets, $V=U_{1} \cup \cdots \cup U_{n}$, and given morphisms $f_{i}: \mathbf{1} \rightarrow \mathbf{1}$ in $\mathcal{K}\left(U_{i}\right)$ such that $f_{i}=f_{j}$ in $\mathcal{K}\left(U_{i} \cap U_{j}\right)$ for $1 \leq i, j \leq n$, there exists a unique morphism $f: \mathbf{1} \rightarrow \mathbf{1}$ in $\mathcal{K}(V)$ such that $f=f_{i}$ in $\mathcal{K}\left(U_{i}\right)$.

Replacing $\mathcal{K}$ by $\mathcal{K}(V)$, we can assume that $V=\operatorname{Spc}(\mathcal{K})$ (see Proposition 1.11). Now, for $n=2$, this is Mayer-Vietoris for morphisms (Corollary 5.8). Note that uniqueness follows from $\operatorname{Hom}_{\mathcal{K}_{\left(U_{1} \cap U_{2}\right)}}(T \mathbf{1}, \mathbf{1})=0$. The induction on $n$ is then easy: To construct $f$, glue the $n-1$ first morphisms $f_{i}$ into a morphism $g: \mathbf{1} \rightarrow \mathbf{1}$ in $\mathcal{K}\left(U_{1} \cup \cdots \cup U_{n-1}\right)$ and show that it agrees with $f_{n}$ in $\mathcal{K}\left(\left(U_{1} \cup \cdots \cup U_{n-1}\right) \cap U_{n}\right)$ - this uses uniqueness for $n-1$; then apply the $n=2$ case to glue $g$ and $f_{n}$ into a global $f$. To prove uniqueness of $f$, proceed similarly, using uniqueness for $n-1$ and for $n=2$ again. Hence the Claim.

Then the existence of the sheaf $\mathbb{G}_{\mathrm{m}}$ is immediate from the claim and from the fact that quasi-compact open subsets form a basis of the topology of $\operatorname{Spc}(\mathcal{K})$ by [1, Remark 2.7 and Proposition 2.14]. For the same reason and because of quasi-compactness of $\operatorname{Spc}(\mathcal{K})$, to define the homomorphism $\alpha$, it suffices to consider $\mathbb{G}_{\mathrm{m}}$-cocycles over finite covers of $\operatorname{Spc}(\mathcal{K})$ by quasi-compact open subsets. In this situation, the gluing is guaranteed by Theorem 5.13. Hence $\alpha$ is well defined.

Finally, injectivity of $\alpha$ is easy. Indeed, given a $\mathbb{G}_{\mathrm{m}}$-cocyle $\sigma$ over a cover $\operatorname{Spc}(\mathcal{K})=U_{1} \cup \cdots \cup U_{n}$ with every $U_{i}$ quasi-compact open, the gluing $a \in \operatorname{Pic}(\mathcal{K})$ comes with isomorphisms $f_{i}: a \stackrel{\sim}{\rightarrow} \mathbf{1}$ in each $\mathcal{K}\left(U_{i}\right)$, compatible with the $\sigma\left(U_{i} \cap U_{j}\right)$ as usual. Now, if $a=\mathbf{1}$, the latter compatibilty means 
that the Čech boundary of the 0-cochain defined by the $f_{i} \in \mathbb{G}_{\mathrm{m}}\left(U_{i}\right)$ is nothing but $\sigma$, that is, $\sigma=0$ in $\check{H}^{1}\left(\operatorname{Spc}(\mathcal{K}), \mathbb{G}_{\mathrm{m}}\right)$.

REMARK 6.9 Note that the condition $\operatorname{Hom}_{\mathcal{K}(U)}(T \mathbf{1}, \mathbf{1})=0$ does not hold in general, for instance in modular representation theory, that is, for $\mathcal{K}=k G-$ stab. For instance, for $k=\mathbb{F}_{2}$ and $G=\mathbb{Z} / 2$, we even have $T \mathbf{1} \simeq \mathbf{1}$.

REMARK 6.10 When the condition $\operatorname{Hom}_{\mathcal{K}(U)}(T \mathbf{1}, \mathbf{1})=0$ holds for every quasi-compact open $U \subset$ $\operatorname{Spc}(\mathcal{K})$ and when $\operatorname{Spc}(\mathcal{K})$ happens to be a scheme, Theorem 6.8 gives an injective homomorphism $\operatorname{Pic}(\operatorname{Spc}(\mathcal{K})) \hookrightarrow \operatorname{Pic}(\mathcal{K})$. In the case of $\mathcal{K}=\mathrm{D}^{\text {perf }}(X)$ for $X$ a scheme, this homomorphism is the one of Proposition 6.4.

\section{Excision}

For later use, we state the next result in greater generality than in section 0. See Remark 5.3. In the following statement, the reader can as well consider the case of $A$ and $B$ reduced to a singleton, that is, $U$ open and $Y$ closed.

THEOREM 7.1 (Excision) Let $\mathcal{K}$ be an idempotent complete strongly closed tensor triangulated category and let $Y \subset U \subset \operatorname{Spc}(\mathcal{K})$. Assume that $Y=\cup_{\alpha \in A} Y_{\alpha}$ with every $Y_{\alpha}$ closed with quasi-compact complement and assume that $U=\cap_{\beta \in B} U_{\beta}$ with every $U_{\beta}$ open and quasi-compact. Then the restriction functor $\rho: \mathcal{K} \rightarrow \mathcal{K}(U)$ induces an equivalence between the respective subcategories of objects supported on $Y$ :

$$
\mathcal{K}_{Y} \stackrel{\sim}{\longrightarrow} \mathcal{K}(U)_{Y}
$$

Proof. Remark first of all that $\operatorname{Spc}(\mathcal{K}(U)) \cong U$ by Proposition 1.11 (stated for $A=\{*\}$ but whose proof generalizes verbatim to the present situation).

Let us see that the functor $\rho: \mathcal{K}_{Y} \rightarrow \mathcal{K}(U)_{Y}$ is full. Given $a, b \in \mathcal{K}_{Y}$ and a fraction $a \stackrel{s}{\longleftarrow} x \stackrel{f}{\longrightarrow} b$ with $s$ a $U$-isomorphism, we have $\operatorname{supp}(\operatorname{cone}(s)) \cap \operatorname{supp}(T a) \subset$ $\operatorname{supp}(\operatorname{cone}(s)) \cap U=\varnothing$, so $\operatorname{Hom}_{\mathcal{K}}(a$, cone $(s))=0$ by Proposition 1.6. So, any distinguished triangle starting with the morphism $s$ must have zero in second place, that is, the morphism $s$ is a split epimorphism, say $s \circ u=\operatorname{id}_{a}$ for some morphism $u: a \rightarrow x$. Amplifying the fraction $f s^{-1}$ by $u$ shows that this morphism $f s^{-1}$ is equal to (the restriction of) the morphism $f u: a \rightarrow b$.

Let us see that the functor $\rho: \mathcal{K}_{Y} \rightarrow \mathcal{K}(U)_{Y}$ is faithful. Let $f: a \rightarrow b$ be a morphism in $\mathcal{K}_{Y}$ such that $\rho(f)=0$, that is, there exists a $U$-isomorphism $s: x \rightarrow a$ such that $f s=0$. As above, $s$ must be a split epimorphism, hence $f=0$.

Let us see that the functor $\rho: \mathcal{K}_{Y} \rightarrow \mathcal{K}(U)_{Y}$ is essentially surjective. Let $b \in \mathcal{K}(U)_{Y}$. There is an object $a \in \mathcal{K}$ such that $\rho(a)=b \oplus T(b)$. We have $\operatorname{supp}(a) \cap U=\operatorname{supp}_{U}(b) \cup \operatorname{supp}_{U}(T b)=$ $\operatorname{supp}_{U}(b) \subset Y$. So, if we call $Z=\operatorname{Spc}(\mathcal{K}) \backslash U$ the complement of $U$, we have proved that $\operatorname{supp}(a) \subset$ $Y \cup Z$. Since $Y \cap Z=\varnothing$, we know by Theorem 1.8 that $a \simeq c \oplus d$ with $\operatorname{supp}(c) \subset Y$ and $\operatorname{supp}(d) \subset$ $Z$. But then $\rho(a)=\rho(c)$ and we have found an object $c \in \mathcal{K}_{Y}$ such that $\rho(c)=b \oplus T(b)$. Now, consider the idempotent of $b \oplus T(b)$ corresponding to the projection on $b$. Since $\rho$ is fully faithful, there exists a corresponding idempotent on the object $c$, which then decomposes accordingly, one factor going to $b$, as was to be shown. 
REMARK 7.2 As an exercise, one can reformulate and prove Excision in the framework of formal Mayer-Vietoris covers (Definition 2.1).

REMARK 7.3 If needed, the reader can establish the following assertion: given a point $\mathcal{P} \in \operatorname{Spc}(\mathcal{K})$, the 'local' category $\mathcal{K} / \mathcal{P}$, or rather its idempotent completion, is equivalent to the colimit of the categories $\mathcal{K}(U)$, over the quasi-compact open neighborhoods $U \ni \mathcal{P}$.

\section{Acknowledgements}

We thank Jacques Thévenaz for a counter-example, which prevented us from losing too much time on some wild dream of ours. The second author thanks the FIM of the Mathematics Department at the ETH Zurich for impartial partial support. We thank the referee for requesting the generalizations to non-tensorial situations.

\section{References}

1. P. Balmer, The spectrum of prime ideals in tensor triangulated categories, J. Reine Angew. Math. 588 (2005), 149-168.

2. P. Balmer, Supports and filtrations in algebraic geometry and modular representation theory, Amer. J. Math. (preprint, 2005), Amer. J. Math. 129 no. 5 (2007).

3. P. Balmer, D. J. Benson and J. F. Carlson, Gluing idempotent modules to make endotrivial modules, Preprint, 2006.

4. P. Balmer and M. Schlichting, Idempotent completion of triangulated categories, J. Algebra 236 no. 2 (2001), 819-834.

5. J. F. Carlson, Constructing endotrivial modules, J. Pure Appl. Algebra, 206 nos. 1-2 (2006), pp. 83-110.

6. H. Fausk, Picard groups of derived categories, J. Pure Appl. Algebra, 180 no. 3 (2003), 251-261.

7. M. Hovey, J. H. Palmieri and N. P. Strickland, Axiomatic stable homotopy theory, Mem. Amer. Math. Soc., 128 no. 610 (1997), x+114.

8. A. Neeman, Triangulated categories, Annals of Mathematics Studies, Vol. 148, Princeton University Press, Princeton, NJ, 2001, viii+449.

9. R. W. Thomason, The classification of triangulated subcategories, Compositio Math. 105 no. 1 (1997), pp. 1-27.

10. R. W. Thomason and T. Trobaugh, Higher algebraic $K$-theory of schemes and of derived categories, The Grothendieck Festschrift, Vol. III, Progress in Mathematics 88, Birkhäuser, Boston, MA, 1990, 247-435.

11. J.-L. Verdier, Des catégories dérivées des catégories abéliennes, Astérisque, 239:xii+253 pp. (1997), 1996. 